\begin{tabular}{|l|l|l|l|l|l|}
\hline MUNIBE Antropologia-Arkeologia & $n^{\circ} 68$ & $155-177$ & DONOSTIA & 2017 & ISSN $1132-2217 \cdot$ elSSN $2172-4555$ \\
\hline
\end{tabular}

\title{
Fases y territorios de la neolitización del NE de la Península Ibérica ca. 5600-4900 cal BC
}

\author{
Periods and territories of the Neolithisation \\ process in NE Iberia ca. 5600-4900 cal BC
}

PALABRAS CLAVES: Neolitizaciòn, cerámica impresa, ${ }^{14} \mathrm{C}$, NE de la Península Ibérica.

GAKO-HITZAK: Neolitizazioa, zeramika inprimatua, ${ }^{14} \mathrm{C}$, Iberiar penintsularen ipar-ekialdea.

KEY WORDS: Neolithisation, impressed pottery, ${ }^{14} \mathrm{C}$, NE Iberian Peninsua..

F. Xavier OMS ARIAS(1)

\section{RESUMEN}

En este trabajo se presentan los datos radiocarbónicos y de las cerámicas decoradas de numerosos yacimientos del NE Peninsular pertenecientes al Neolítico antiguo (c.5600-4900). Mediante el análisis de los dos registros, se han podido detectar características culturales independientes en diferentes territorios de esta región. Después de una primera ocupación de poca entidad, probablemente relacionada con el Neolítico Impressa, se pasa a una primera oleada de poblaciones cardiales. Éstas se distribuyen por diferentes ámbitos geográficos litorales y prelitorales y ya presentan diferencias en sus producciones cerámicas. Durante una fase evolucionada (a partir de c. 5300 cal BC), los territorios diversifican sus producciones cerámicas aún más en los territorios litorales y prelitorales. Sin embargo, las nuevas zonas ocupadas presentan registros donde se mezclan los componentes cardiales y los epicardiales, sobretodo en la región de Segre-Pirineos. Estos datos reflejan por un lado una rápida distribución por todo el territorio durante la neolitización, pero también un carácter particular entre diferentes comunidades neolíticas, lejos de una visión homogénea del proceso.

\section{LABURPENA}

Lan honetan, penintsularen ipar-ekialdeko aztarnategi askotan aurkitutako Neolito zaharreko (c. 5600-400) datu erradiokarbonikoak eta zeramika apainduenak aurkeztu ditugu. Bi erregistroak aztertuta eremu horretako lurralde batzuetako eta besteetako ezaugarri kultural independenteak detektatu ahal izan dira. Seguru asko Neolito Inprimatuarekin lotutako garrantzi txikiko lehen okupazioaren ondoren, lehen populazio kardialen olatua iritsi zen. Itsasertzeko eta itsasertz aurreko zenbait gune geografikotan banatuta daude eta aldeak daude zeramikazko ekoizpen-lanetan. Fase eboluzionatuan (c. 5300 cal BC-tik aurrera), lurraldeek are gehiago dibertsifikatu zituzten zeramikazko ekoizpen-lanak itsasertzeko eta itsasertz aurreko lurraldeetan. Hala ere, okupatutako eremu berrietan erregistroak daude eta horietan nahasi egiten dira osagai kardialak eta epikardialak, batez ere Segre-Pirinioak eskualdean. Datu horiek erakusten dute, batetik, neolitizazioan azkarra izan zela banaketa lurralde osoan, eta horrez gain, komunitate neolitikoen artean, prozesuaren ikuspegi homogenoa izatetik urrun, izaera partikularra zegoela.

\section{ABSTRACT}

In this paper we present the radiocarbon and decorated pottery data from a several sites of NE Iberia, belonging to Eary Neolithic (5600$4900 \mathrm{cal} \mathrm{BC}$ ). In a first step, we have worked with radiocarbon data available. Initially, a quality filter have been applied to reach a 53 optimal-quality dates. Through Cluster (Euclidean), Sum of Probabilities and Bayesian analyzes, four chronological phases have been stabblished. In a second step, pottery analyzes has carried out on 21 sites, located on 8 different regions.

The four chronological phases (Phase 0 c. 5620-5470 cal BC; Phase 1 c. 5480-5320 cal BC; Phase 2, c. 5350-5010 cal BC; Phase 3, 5050-4800 cal BC) do not correspond clearly with the characteristics of decorated potteries. So we have determined the existence of just two chronological and cultural periods. The early stage of the first period (from $5650 \mathrm{cal} \mathrm{BC}$ ) is exemplified by just two radiocarbon dates -from Guixeres de Vilobí and El Cavet- and a cluster of potteries from these two sites have been identified. Ligurian-Impressa pottery type is not clearly represented in these sites. From c. 5500 cal BC, Cardial type pottery is the most spread. Sites are located on coastal (Tarragona group) and precoastal areas (Vallès and Penedès groups). The pottery decoration indicates a certain homogeneity. but also some specific decorative techniques to each region, exclusive of others.

During the second periode (c.5300-4900 cal BC), Cardial type pottery is still present in the same territories of the previous phase, in coastal and precoastal territories. However, in the hinterland areas (Pyrenean and Prepyrinean ranges), other pottery style is attested, based in other kind of impressions, incisons, even the boquique technique; Cardial use was scarce. A mixture between Cardial traditions and other techniques was performed in other areas, as inner Tarragona and Ebro.

This deep analysis (filtered radiocarbon data, along with the analysis of pottery decorative traditions) have allowed to get information about the internal evolution and recombination of the first farmers in NE of the Iberian Peninsula. The new data obtained in this study also will enable comparison of our records with neighboring territories, where the information had always been more abundant.

\footnotetext{
(1) SERP, Seminari d'Estudis i Recerques Prehistòriques. Dept. d'Història i Arqueologia. Universitat de Barcelona, C/ Montalegre 6-8, 08001 Barcelona.xavieroms@gmail.com, oms@ub.edu
} 


\section{1.- INTRODUCCIÓN}

El proceso de llegada y consolidación de las sociedades productoras al occidente Mediterráneo es una temática muy extendida en la literatura científica, aunque ha tenido una incidencia desigual dependiendo de la región. Existen territorios como el sur y sureste de Francia (Guilaine, 1976; Manen, 2002; Guilaine, 2007; Guilaine \& Manen, 2007), Valencia (Bernabéu, 1989, 1999; Martí, 1977, 2008), Andalucía (Navarrete, 1976; García Borja et al., 2014) y Portugal (Carvalho, 2008, 2010; Zilhão, 2001; 2004) donde la tradición investigadora ha sido importante. Recientemente, otros territorios han sumado importantes aportaciones al panorama peninsular (Rojo et al., 2008; García Martínez de Lagrán, 2014). Por su parte, el NE de la Península Ibérica ha sido considerado en numerosas ocasiones como una región clave para el estudio de la neolitización del Mediterráneo occidental (Guilaine, 1976; Bernabéu, 1999; Zilhão, 2001; Manen, 2002; Martí, 2008; entre otros). Sin embargo, la producción focalizada en este territorio ha sido escasa y reducida a algunas síntesis y monografías puntuales.

Los primeros datos sobre materiales del Neolítico inicial en esta región se remontan a finales del siglo XIX, cuando el geólogo Lluís Marià Vidal exploró algunas cavidades de la zona del Montsec (Prepirineo de Lleida) (Serra Ràfols, 1921). Posteriormente, las publicaciones primero de la Cova de Can Pasqual (Bosch Gimpera, 1915-1920), la Cova Gran y la Cova Freda (Colomines, 1925) y después de l'Esquerda de les Roques del Pany (Grivé, 1936) igualan esta zona a otras regiones medite- rráneas (Guilaine, 1976; Martí, 2008). Después de esa fecha, las publicaciones del Neolítico inicial escasean en Cataluña, y siempre se refieren a pequeñas exavaciones o revisiones de material descontextualizado (Giró, 1947-1948; Colomines, 1947; Vilaseca, 1969; Baldellou, 1972, 1974, 1979).

A mediados de la década de los 70' del siglo XX se inician las primeras campañas por parte de jóvenes arqueólogos que habían excavado en el sur de Francia con Jean Guilaine: Josep Mestres (en colaboración con V. Baldellou) en Les Guixeres de Vilobí y Araceli Martín en la Cova del Frare, yacimientos con importantes paquetes del Neolítico inicial y donde ya se aplicaron métodos de excavación tridimensional. A partir del año 1976, el investigador francés Jean Guilaine desembarcó en Catalunya junto a su equipo en un proyecto codirigido con Miquel Llongueras. Este grupo excavó el yacimiento epipaleolítico de la Balma del Gai y las cavidades con importantes conjuntos neolíticos de la Cova del Toll y la Balma de l'Espluga (Guilaine et al., 1982), aunque los resultados fueron escasamente publicados. Poco después, la celebración y publicación del coloquio de la Taula Rodona de Montserrat sobre "El Neolític a Catalunya" supone un importante impulso para la investigación en el NE peninsular (A.A.V.V., 1981). Por desgracia, desde ese momento, la producción sobre el Neolítico inicial en Cataluña no ha sido numerosa y las monografías, escasas. Las pocas excepciones son la Draga (Bosch et al., 2000; 2011), Plansallosa (Bosch et al., 1998) y Sant Pau del Camp (Molist et al., 2008).

En el NE de la península Ibérica se han identificado hasta la fecha 78 enclaves que han proporcionado ce-

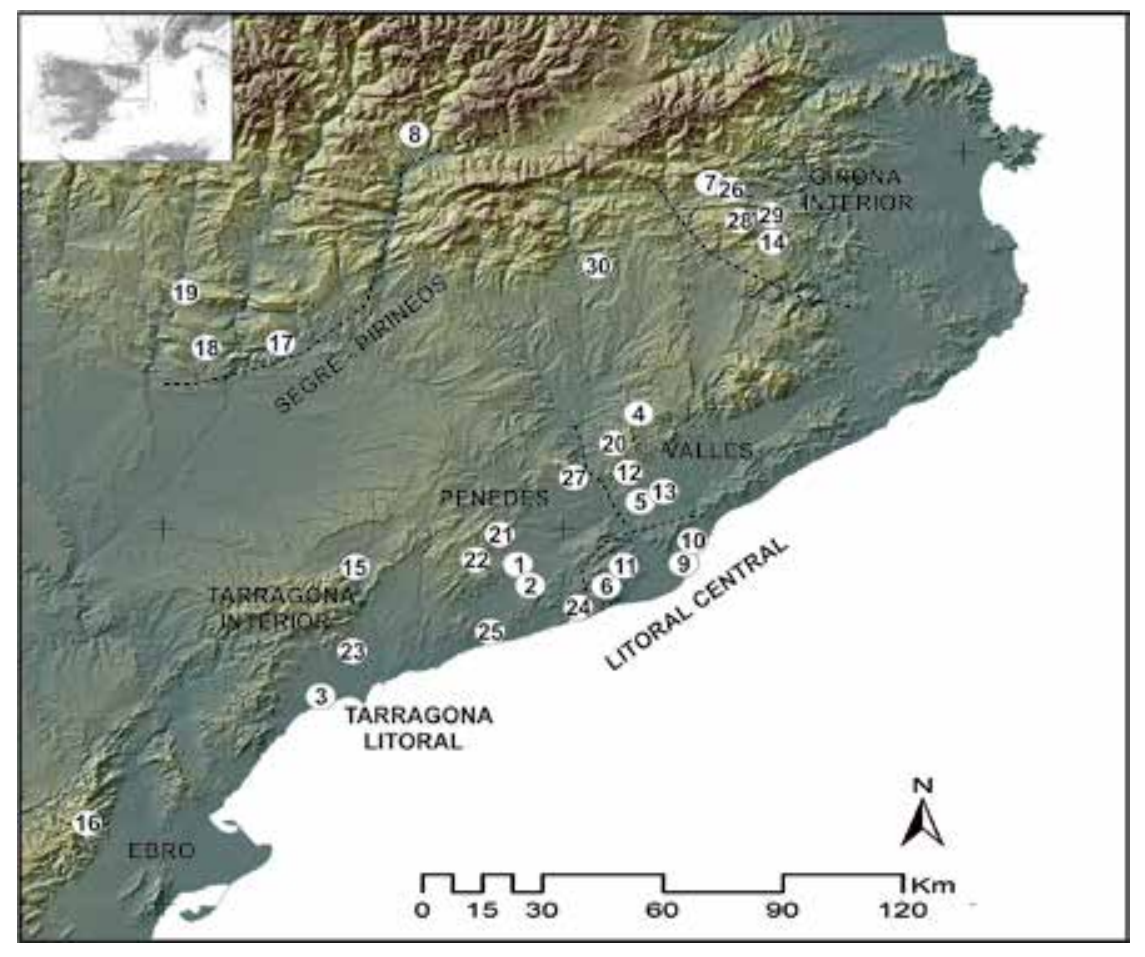

Fig. 1. Territorios y yacimientos nombrados en este trabajo. 1 Guixeres de Vilobí; 2 La Serreta; 3 Cavet; 4 Cova del Toll; 5 Can Roqueta II; 6 Cova de Can Sadurní; 7 Balma del Serrat del Pont; 8 Balma Margineda; 9 Sant Pau del Camp; 10 Plaça Vila de Madrid; 11 Cova Bonica; 12 Cova del Frare; 13 Turó de Can Bellsolà; 14 La Draga; 15 Cova de la Font Major; 16 Cova del Vidre; 17 Cova del Parco; 18 Cova Gran de Santa Linya; 19 Cova Colomera; 20 Balma de l'Espluga; 21 Cova de la Guineu y Cova del Bolet; 22 Esquerda de les Roques del Pany; 23 Coll Blanc; 24 Cova de Sant Llorenç; 25 Cova Foradada; 26 Plansallosa; 27 Cova Gran i Cova Freda; 28 Cova d'en Pau; 29 Cova de l'Arbreda; 30 Font del Ros (Autor base cartográfica: Gerard Remolins) / Sites and territories named in the text. 1 Guixeres de Vilobí; $2 \mathrm{La}$ Serreta; 3 Cavet; 4 Cova del Toll; 5 Can Roqueta II; 6 Cova de Can Sadurní; 7 Balma del Serrat del Pont; 8 Balma Margineda; 9 Sant Pau del Camp; 10 Plaça Vila de Madrid; 11 Cova Bonica; 12 Cova del Frare; 13 Turó de Can Bellsolà; 14 La Draga; 15 Cova de la Font Major; 16 Cova del Vidre; 17 Cova del Parco; 18 Cova Gran de Santa Linya; 19 Cova Colomera; 20 Balma de l'Espluga; 21 Cova de la Guineu y Cova del Bolet; 22 Esquerda de les Roques del Pany; 23 Coll Blanc; 24 Cova de Sant Llorenç; 25 Cova Foradada; 26 Plansallosa; 27 Cova Gran i Cova Freda; 28 Cova d'en Pau; 29 Cova de l'Arbreda; 30 Font del Ros (Base map: Gerard Remolins) 
rámicas impresas pertenecientes a las primeras fases neolíticas (Martín, 1992; Martín \& Vaquer, 1995; Oms, 2014), aunque sólo algunos cuentan con estratigrafía, materiales y dataciones radiocarbónicas. En los últimos años, en Cataluña, se ha llevado a cabo un importante esfuerzo en la consecución de fechas para el lapso c. 5600-4900 cal. Ello ha posibilitado la correlación de los materiales cerámicos, las zonas geográficas y las fechas 14C disponibles. En este artículo se van a sintetizar los resultados más importantes de una reciente investigación (Oms, 2014) y además se han añadido nuevas dataciones, ahora disponibles.

\section{MATERIAL Y MÉTODOS}

\subsection{Las dataciones}

De un total de 68 dataciones radiocarbónicas disponibles, se ha procedido a aplicar una filtro simplificado basado en las propuestas de Bernabéu (2006) y de Zilhão (2011), aplicado recientemente en otros trabajos (Oms et al., 2016c). 15 dataciones han sido rechazadas por superar los 100 años de variación estándar, por presentar horizontes arqueológicos poco claros o poco explicados y por contar con nuevas dataciones -en taxones de vida corta- que sustituyen a las primeras. De las 53 restantes, 13 han sido definidas como de "calidad secundaria" a causa de varias razones: por tratarse de carbones no identificados taxonómicamente, por ser taxones de vida corta no domésticos o carbones de a priori vida larga (ver apéndice). Sin embargo, todas estas dataciones las consideramos válidas por contar con otra/s fecha/s del mismo nivel en taxones de mejor "calidad" y resultar coherentes y por proceder de un contexto antrópico con buena asociación entre resultado radiocarbónico y cultura material. Por último, dos dataciones sobre resto humano proceden de contextos algo problemáticos. Plaça Vila de Madrid no presenta asociación con ningún ítem cultural significativo (Pou et al., 2010) y Cova Foradada no tiene un nivel del Neolítico inicial stricto sensu, aunque si materiales de esa fase mezclados en una capa sepulcral del III milenio (Oms et al., 2016a). En ambos casos y por la naturaleza del material datado, el resultado radiocarbónico per se tiene un valor suficiente para que estas fechas sean usadas.

Las dataciones aceptadas de "calidad primaria" (40) han sido obtenidas sobre muestras de vida corta, en su mayoría domésticas y proceden de contextos fiables. Algunas (4) han sido obtenidas de ramitas de carbón de taxones de una potencial vida corta (o reducida) como Arbutus unedo en La Serreta y la Cova Bonica, Buxus sempervirens en la Cova Colomera y Laurus nobilis en la Cova de la Guineu (tabla 1).

Las dataciones radiocarbónicas han sido calibradas mediante el software OxCal v.4.2.4 (Bronk Ramsey, 2013) y la curva IntCal'13 (Reimer et al., 2013). Cuando se dispone de más de una datación del mismo nivel o estructura, se ha realizado un test $X^{2}$ con el objetivo de determinar la homogeneidad de dichas fechas. Cuando el resultado ha sido positivo se ha realizado una datación media. Estas operaciones han sido realizadas mediante el software Calib 7.0.4. Las dataciones resultantes (44), han sido sometidas a un análisis cluster (realizado mediante el software libre PAST) y a una suma acumulativa y por último a un análisis Bayesiano (Phases Contiguous), realizados nuevamente con OxCal v.4.2.4.

\begin{tabular}{|l|l|l|}
\hline Tipo de muestra & \multicolumn{1}{|c|}{ (A) NR (\%) } & \multicolumn{1}{|c|}{ (B) NR (\%) } \\
\hline Cereal & $19(35,8)$ & $14(31,8)$ \\
\hline Fauna doméstica & $15(28,3)$ & $11(25)$ \\
\hline Fauna salvaje & $1(1,9)$ & $1(2,2)$ \\
\hline Fruto silvestre & $2(3,7)$ & $2(4,5)$ \\
\hline Carbón determinado & $7(13,2)$ & $7(15,9)$ \\
\hline Carbón indeterminado & $7(13,2)$ & $5(11,3)$ \\
\hline Hueso humano & $2(3,7)$ & $2(4,5)$ \\
\hline Mix (semilla y carbón o hueso) & - & $2(4,5)$ \\
\hline Total & $\mathbf{5 3}(\mathbf{1 0 0})$ & $\mathbf{4 4}(\mathbf{1 0 0})$ \\
\hline
\end{tabular}

Tabla 1: Tipos de muestra datada. $A=$ con la totalidad de muestras; $B=$ con las dataciones reducidas por multiplicidad de fechas por nivel $\left(X^{2}\right)$. *En la columna $B$ se contabilizan 44 dataciones puesto que hay dos casos donde se combinan restos diferentes (hueso y semilla o carbón) al realizar el $X^{2}$ de fechas de un mismo nivel o estructura / Type of sample dated. $A=$ with all the samples; $B=$ datings reduced by multiplicity per layer $\left(X^{2}\right)$. * in column $B$ are recorded 44 dating because two different cases combine different remains (bone and seed or charcoal) when $\mathrm{X}^{2}$ is performed.

\subsection{La cerámica}

La cerámica ha sido analizada macroscópicamente realizando un recuento por número máximo de vasos decorados por cada yacimiento. Este tipo de análisis favorece la observación de parámetros como el motivo y la sintaxis decorativa, así como la multiplicidad o no de matrices utilizadas e impide la repetición de elementos de un mismo vaso (Oms, 2014). La identificación de las matrices utilizadas ha sido definida a través de trabajos recientes (Manen \& Salanova, 2010) y los motivos, a falta de recipientes completos, han sido definidos a partir del modelo simplificado de Claire Manen (2002). Nuestro estudio se ha realizado sobre un total de 809 vasos cerámicos. Sin embargo, el hecho de que la Balma del Serrat del Pont III.4 y la Balma Margineda c3b tengan sólo 3 y 4 vasos respectivamente ha provocado que el estudio decorativo se realice finalmente sobre 802 vasos decorados.

La alta fragmentación del registro ha impedido, en muchas ocasiones, poder definir la totalidad de los motivos decorativos. Por ese motivo, en los recuentos totales, los datos pueden variar del número total de efectivos disponibles para cada yacimiento/territorio en cuanto a las matrices decorativas y los motivos.

Para contar con un mayor número de yacimientos, se ha analizado el material de yacimientos sin excavación arqueoestratigráfica pero que cuentan con un horizonte aparentemente homogéneo y relativamente numeroso: Esquerda de les Roques del Pany, Cova del Bolet y Balma de l'Espluga. 
En la problemática entre datación-registro cerámico, destaca el silo 2014 del Cavet. Éste, con una datación sobre semilla de $6535 \pm 36 \mathrm{BP}$, cuenta con escasos fragmentos cerámicos de los cuales sólo uno presenta una decoración, un borde recto con labio plano impreso (con instrumento). Este hecho dificulta la correlación de esta datación con el resto del yacimiento. En este mismo sentido, Les Guixeres cuenta con una datación que resulta elevada para conjuntos cardiales (OxA-26068) (Oms et al., 2014; Martins et al., 2015). De entre los vasos analizados existe un pequeño conjunto que podría pertenecer a una fase Impressa. Por ese motivo, tanto la datación como los materiales nombrados van a ser tratados de manera separada, en la discusión. Para la Cova del Parco, con una datación de un silo al que sólo se le asocian restos de un vaso decorado (Petit, 1996), se le han añadido el resto de materiales pertenecientes a las campañas de Maluquer de Motes. Consideramos válida esta asociación puesto que recientemente, en el vecino yacimiento Abric del Xicotó, las dataciones disponibles y el material cerámico decorado son claramente análogos y homogéneos con el conjunto de Parco (X. Mangado com. pers.).

Por último, para obtener una mayor representatividad y una visión de conjunto más amplia, han sido unidos los conjuntos cerámicos de un mismo territorio natural y más cercanos a nivel cronológico. Estos "grupos geográficos" y las fases serán analizados en el resto del trabajo.

\section{RESULTADOS}

\subsection{El marco cronológico}

Después de la reducción de dataciones homogéneas del mismo nivel ( $v$. apéndice), se ha obtenido una horquilla general ca. 5600-4800 cal BC a 20. Ésta es parecida a la de otros espacios del Neolítico inicial del Mediterráneo occidental, como el sur de Francia (Manen \& Sabatier, 2003), el levante español (Bernabéu, 2006; Bernabéu et al., 2009) y Portugal (Zilhão, 2001; Carvalho, 2010).

En primer lugar hemos sometido las dataciones a un análisis cluster (tipo Euclidean). Utilizando los extremos de la horquilla a $2 \sigma$, este método ha establecido la presencia de 4 momentos diferencidados a nivel cronológico, que hemos denominado fases (fig. 2.A). Éstas ven correctamente representado su desarrollo en las sumas de probabilidad (fig. 2.B). Para testar definitivamente este modelo se ha llevado a cabo un análisis Bayesiano (de 4 fases contiguas), que ha proporcionado una validez notable: $A_{\text {model }}=129.7 / A_{\text {overall }}=129.8$ (fig. 3). El esquema resultante sobre las fases cronológicas es el siguiente:

- El primero, Fase 0, está formado por sólo dos dataciones, de la base de Les Guixeres de Vilobí y del silo 2014 de el Cavet. Se sitúa entre 5620-5470 cal BC.

- Una agrupación ca. 5480-5320 cal BC, Fase 1, con la otra datación de Les Guixeres (OxA-26069), Cova del Toll 2b, Cova de Can Sadurní c18, Can Roqueta II CR-
173, La Serreta (todos los silos), Balma del Serrat del Pont III.4, Plaça Vila de Madrid, Balma Margineda 3b y el silo NE36 de la Font del Ros.

- Una tercera, Fase 2, formada por numerosas dataciones situadas ca. 5350-5010 cal BC, entre las que se encuentran las de la Cova del Vidre II y II-hogar, Colomera CV10, CE13 y CE14, Sant Pau del Camp s.1 y s.2, Cova de la Font Major Ig, Cova Bonica IVe, Turó de Can Bellsolà TCB-4, Font del Ros NE15 y NE33, Cova del Frare c6, Cova Foradada lb base, Draga sectores A, B y C, Cova de Sant Llorenç n2 y Cova de la Guineu le.

- Por último, la Fase 3 se desarrolla entre ca. 50504800 cal BC. Estaría compuesta por las dataciones de la Draga E56 y E3, Cova Gran E9, Cova Colomera CE12, Coll Blanc, Cova del Parco EE1, Balma Margineda C3F y Font del Ros NE21.

A pesar de la existencia de 4 unidades a nivel cronológico, se ha optado por aislar la Fase 0 con un reducido conjunto de materiales cerámicos asociados. La Fase 1 cuenta con las dataciones y materiales de los yacimientos antes definidos. Por su parte, las fases 2 y 3 han ido unificadas en sólo una, ca. 5300-4800 cal $\mathrm{BC}$, ya que los datos culturales de las fases tercera y cuarta, dentro de un mismo yacimiento (casos de Draga y Colomera) son totalmente homogéneos y no se ha considerado representativo mantenerlos separados. El análisis Bayesiano de esta unión certifica que la horquilla es homogénea y válida $\left(A_{\text {model }}=86.7\right.$ y $\left.A_{\text {overall }}=76.4\right)$.

Para profundizar en nuestro estudio, hemos situado cada yacimiento del que tenemos datos cerámicos en una Fase cronológica. En la tabla 2 se resume la cantidad de material de los yacimientos, la región natural a la cual pertenecen y la fase en que se integran. Asimismo, se unen aquellos registros de una misma zona y periodo. Esta es la nomenclatura que se va a usar en los siguientes apartados, destinados a caracterizar las producciones cerámicas del NE peninsular.

\subsection{La cerámica}

\subsubsection{Técnicas decorativas simplificadas}

A nivel de matrices decorativas, en la Fase 1 se observa cierta homogeneidad entre Penedès 1 y Vallès 1 , claramente diferenciados de el Cavet. En los primeros se da una relativa pobreza en las técnicas utilizadas: el cardial como única matriz está presente en c. $60 \%$ de los vasos; los cordones lisos serían la segunda técnica más utilizada, con aproximadamente un $20 \%$. Las impresiones de instrumento (sencillo o peine) tienen un peso desigual, en Penedès es casi inexistente mientras que en Vallès 1 se da en 4 vasos (6,8\%). La incisión/acanalado tiene un peso irrelevante, con sólo un vaso en Vallès 1. La combinación de técnicas es rara y poco numerosa. De éstas, la más habitual es el cardial sobre cordón, presente en cerca de un $10 \%$ de los dos registros. A más distancia se documenta la impresión de instrumento sobre cordón $(<2 \%)$ o la impresión cardial combinada con 


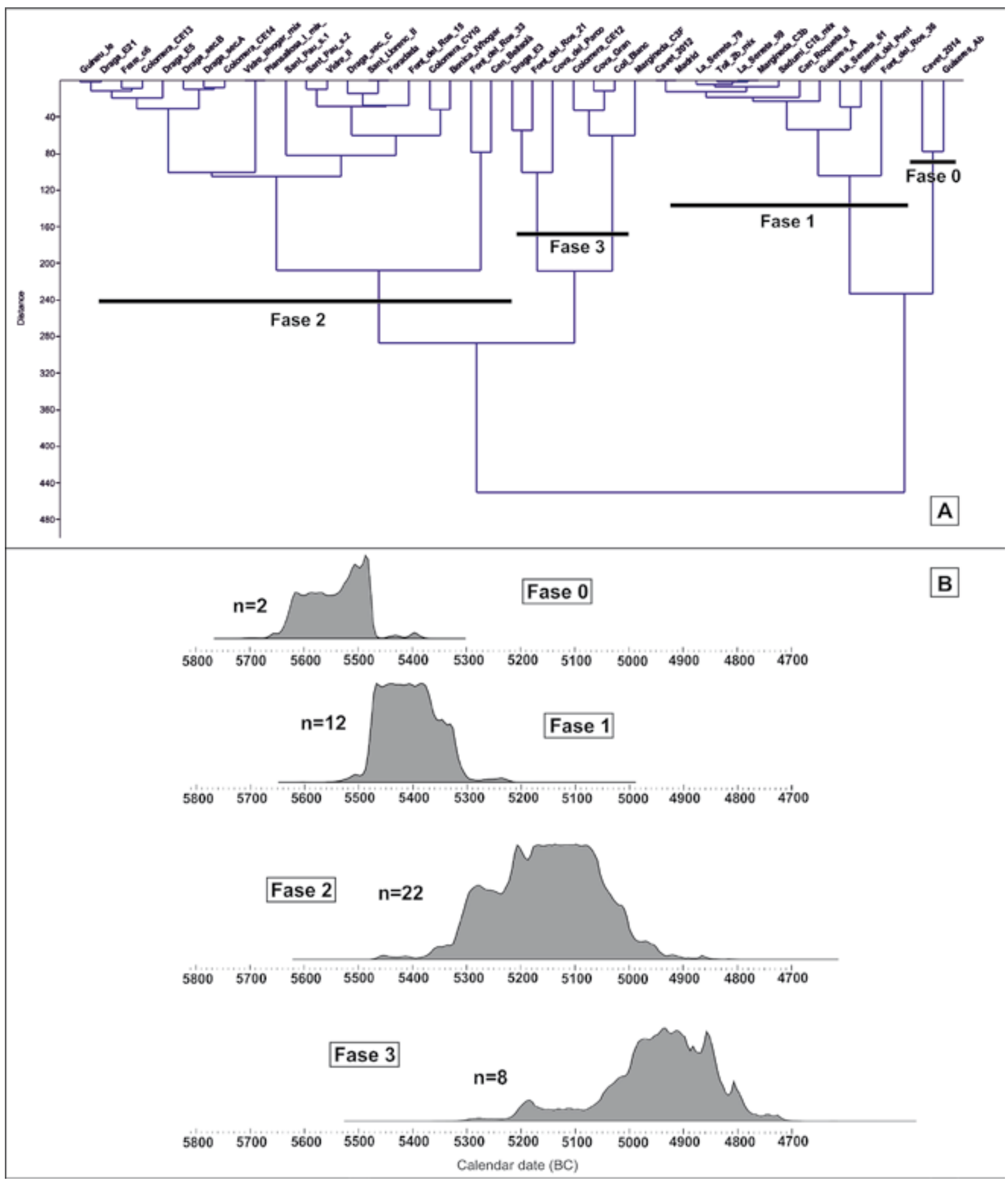

Fig. 2. A, Cluster de dataciones (software PAST); B, Gráfico acumulativo de las dataciones disponibles (software OxCal v.4.2). En ambos casos se señalan las fases cronológicas / A, Cluster of dates (softare PAST); B, Cumulative chart available datings (software OxCal v.4.2). In both cases the chronological phases are identified.

incisiones o impresiones de instrumento con 3 vasos en cada caso. El Cavet supone un caso distinto a Penedès 1 y Vallès 1. La impresión cardial y el cordón liso tienen la misma presencia (30\%), la impresión de instrumento y la incisión están presentes en 2 y 3 vasos respectivamente. El boquique está presente en un solo vaso. Las combinaciones de técnicas son solamente el cardial sobre cordón y la impresión de instrumento con cardial. 


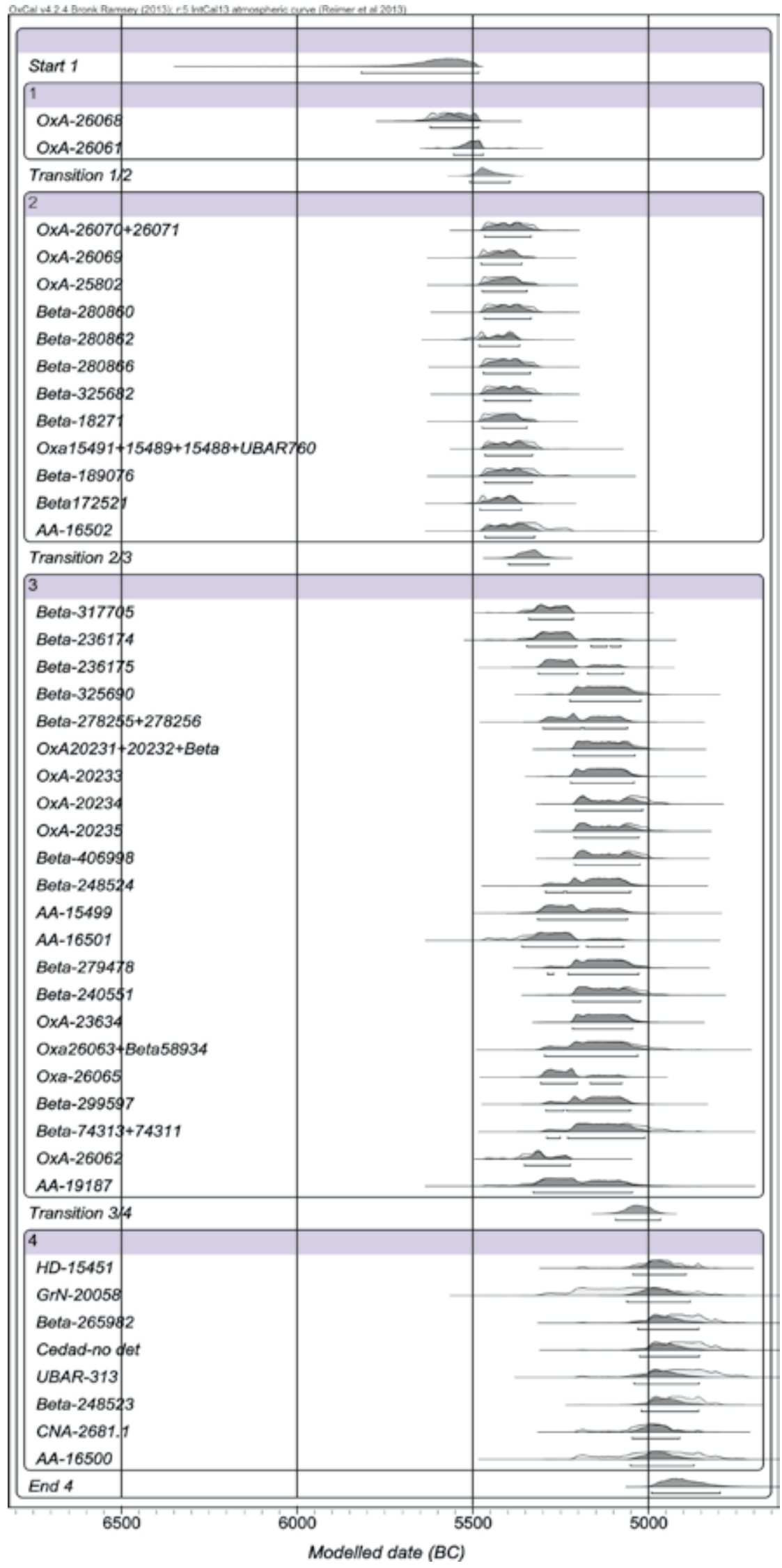

Fig. 3. Análsisi bayesiano (de fases contiguas) de las 4 fases cronológicas, realizado mediante OxCal v.4.2 / Bayesian analyses (Phase contiguous) of the 4 chronological phases, performed using OxCal v.4.2. 


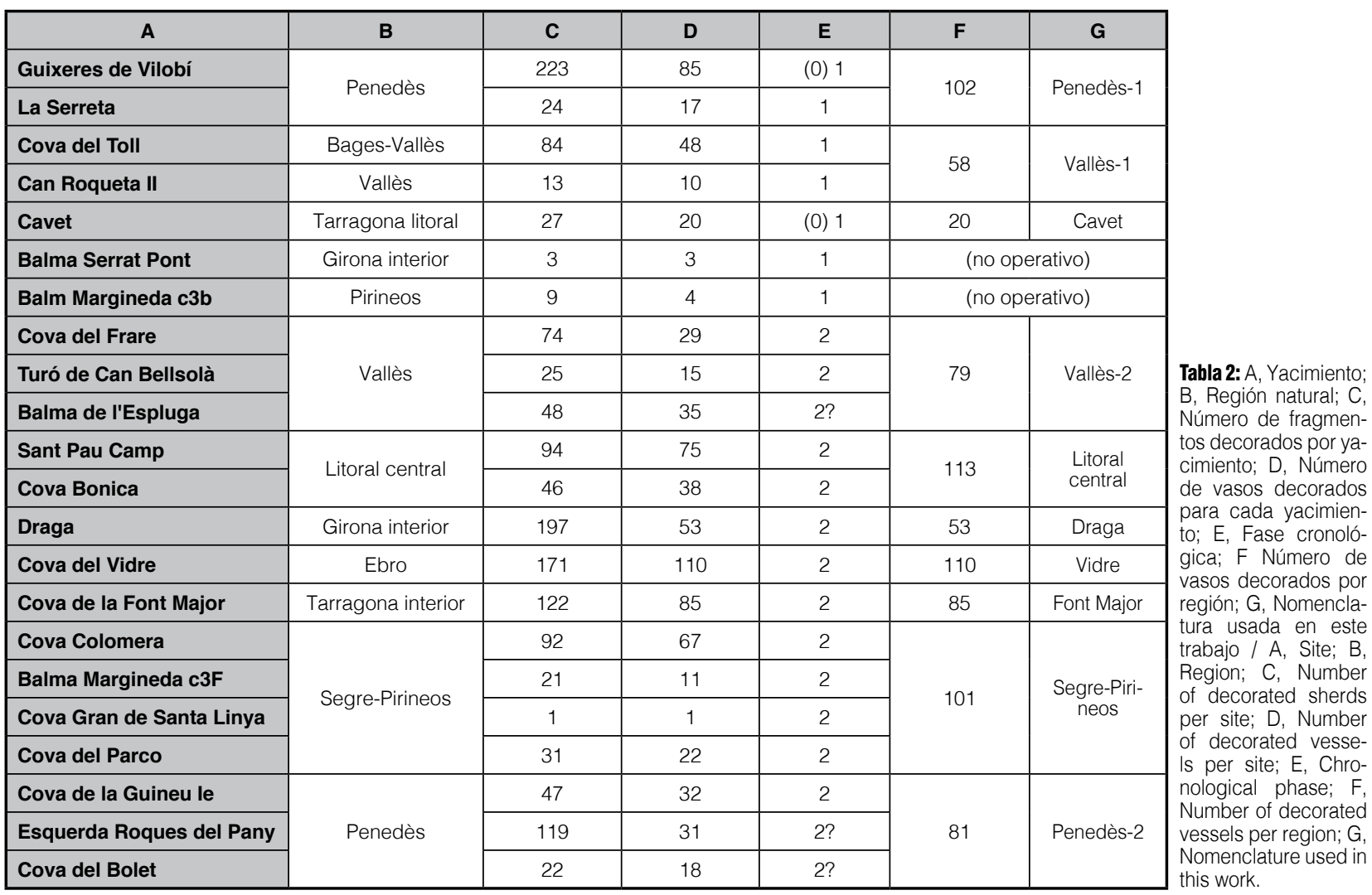

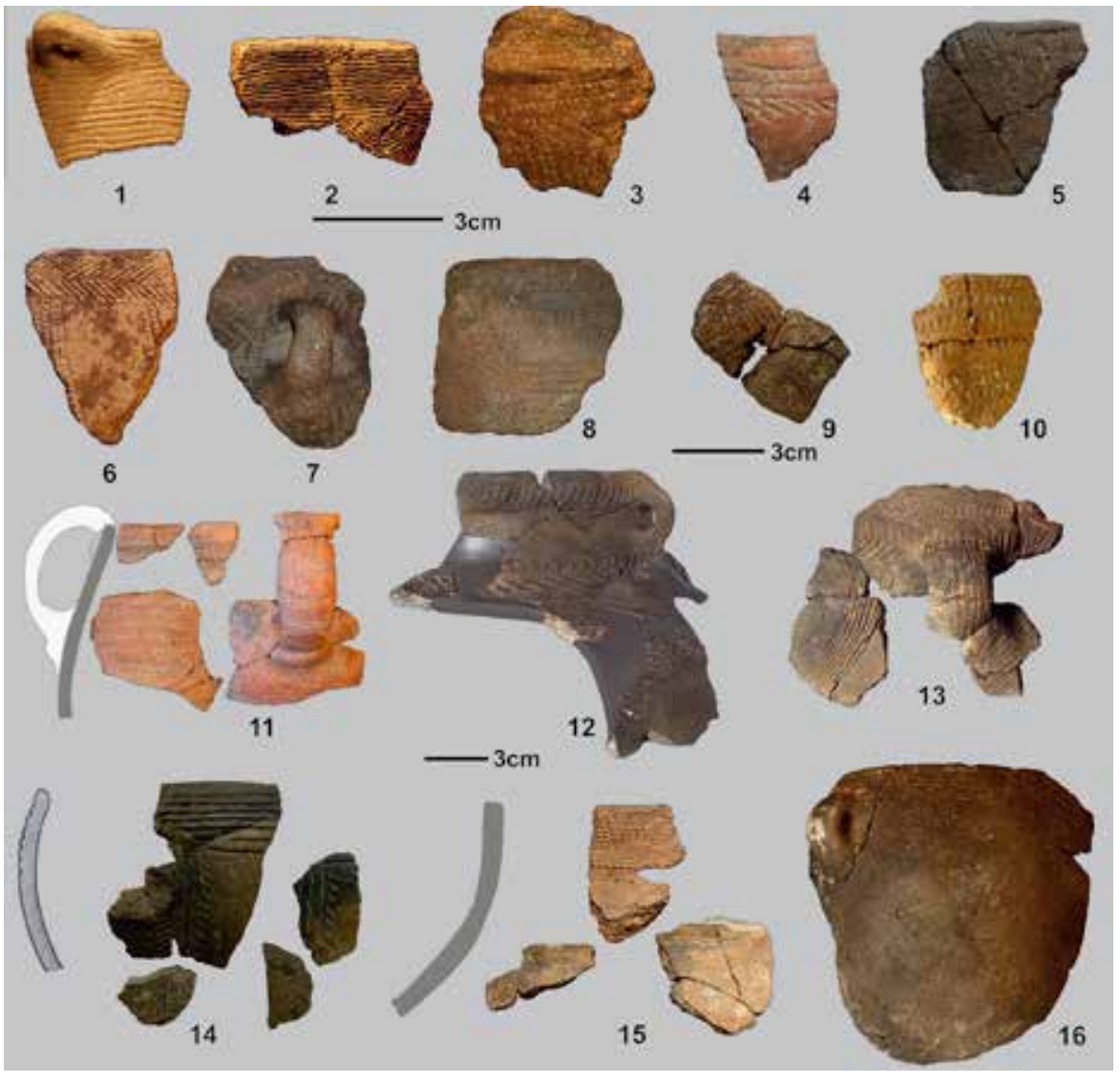

Fig. 4. Ejemplos de cerámicas decoradas de la Fase 1: La Serreta (1-3), Guixeres de Vilobí $(4,11,15,16)$, Cavet $(5,9,10)$, Cova del Toll 6-8), Cova de Can Sadurní $(12,13)$ y Balma Margineda (14). Las imágenes de Can Sadurní proceden de Blasco et al., 2009 / Decorated potteries from the Phase 1: La Serreta (1-3), Guixeres de Vilobí $(4,11,15,16)$ Cavet $(5,9,10)$, Cova del Toll $(6-8)$, Cova de Can Sadurní $(12,13)$ y Balma Margineda 14). Cova de Can Sadurní potteries proceed from Blasco et al., 2009. 


\begin{tabular}{|c|c|c|c|c|c|c|c|c|c|c|c|}
\hline & A & B & C & D & E & $\mathbf{F}$ & G & $\mathbf{H}$ & I & $\mathbf{J}$ & $\mathbf{K}$ \\
\hline \multicolumn{12}{|c|}{ Datos totales } \\
\hline Cavet & 6 & 1 & 2 & 3 & 6 & 0 & 1 & 0 & 1 & 0 & 20 \\
\hline Penedès 1 & 65 & 10 & 1 & 0 & 21 & 2 & 3 & 0 & 0 & 0 & 102 \\
\hline Vallès 1 & 34 & 5 & 4 & 1 & 10 & 1 & 3 & 0 & 0 & 0 & 58 \\
\hline Penedès 2 & 25 & 20 & 5 & 1 & 17 & 4 & 7 & 2 & 0 & 0 & 81 \\
\hline Vallès 2 & 27 & 4 & 10 & 1 & 20 & 3 & 14 & 0 & 0 & 0 & 79 \\
\hline Litoral central & 59 & 12 & 8 & 4 & 20 & 5 & 2 & 0 & 3 & 0 & 113 \\
\hline Segre-Pirineos & 0 & 2 & 36 & 14 & 15 & 11 & 5 & 5 & 9 & 4 & 101 \\
\hline Vidre & 15 & 1 & 17 & 11 & 17 & 12 & 16 & 19 & 2 & 0 & 110 \\
\hline Draga & 14 & 5 & 5 & 0 & 10 & 0 & 19 & 0 & 0 & 0 & 53 \\
\hline Font Major & 23 & 13 & 10 & 2 & 22 & 6 & 4 & 3 & 2 & 0 & 85 \\
\hline Total & 268 & 73 & 98 & 37 & 158 & 44 & 74 & 29 & 17 & 4 & 802 \\
\hline \multicolumn{12}{|c|}{ Datos porcentuales } \\
\hline Cavet & 30 & 5 & 10 & 15 & 30 & 0 & 5 & 0 & 5 & 0 & 100 \\
\hline Penedès 1 & 63,7 & 9,8 & 0,9 & 0 & 20,5 & 1,8 & 2,9 & 0 & 0 & 0 & 100 \\
\hline Vallès 1 & 58,6 & 8,6 & 6,8 & 1,72 & 17,2 & 1,7 & 5,1 & 0 & 0 & 0 & 100 \\
\hline Penedès 2 & 30,8 & 24,7 & 6,2 & 1,2 & 20,9 & 4,9 & 8,6 & 2,4 & 0 & 0 & 100 \\
\hline Vallès 2 & 34,17 & 5 & 12,6 & 1,2 & 25,3 & 3,7 & 17,7 & 0 & 0 & 0 & 100 \\
\hline Litoral central & 52,2 & 10,6 & 7 & 3,5 & 17,7 & 4,4 & 1,7 & 0 & 2,6 & 0 & 100 \\
\hline Segre-Pirineos & 0 & 1,9 & 35,6 & 13,8 & 16,8 & 10,9 & 4,9 & 4,9 & 8,9 & 3,9 & 100 \\
\hline Vidre & 13,1 & 0,87 & 14,9 & 9,6 & 14 & 10,5 & 16 & 16,6 & 1,7 & 0 & 100 \\
\hline Draga & 23,7 & 8,4 & 8,46 & 0 & 16,9 & 0 & 35,5 & 1,7 & 0 & 0 & 100 \\
\hline Font Major & 27 & 15,3 & 11,7 & 2,3 & 25,8 & 7 & 4,7 & 3,5 & 2,3 & 0 & 100 \\
\hline
\end{tabular}

Tabla 3: Decoraciones simplificadas: A Cardial; B Cardial sobre cordón; C Impresión-peine; D Incisión/acanalado; E Cordón liso; F Cordón impreso/inciso; G Cardial con incisión o impresión; H Impresión con incisión; I Boquique; J Boquique con impresión o incisión; K Total / Simplified decoration techniques: A, Cardial; B, Cardial on cord; C, Impressed; D Incision; E Plain cord; F Impressed or incised cord; G Cardial with impressed or incised; H Impressed and incised; I Boquique; J Boquique with impresión or incision; K Total.

Durante la Fase 2, todos los yacimientos aumentan la importancia de las combinaciones decorativas. Los porcentajes de cardial disminuyen sensiblemente en VaIlès 2 y Penedès 2, con porcentajes entre 25-35\%. Esta misma presencia se mantiene en la Draga y la Cova de la Font Major, pero es mucho menor en la Cova del Vidre y sobretodo en el grupo Segre-Pirineos. Los cordones lisos tienen una presencia muy equilibrada en todos los grupos, entre 15-25\%, y solamente en esta fase se dan los de tipo interno en el labio. Siguiendo en las matrices únicas, las impresiones simples y de peine tienen un peso desigual entre los yacimientos. De ser una técnica predominante en Segre-Pirineos y abundante en Vidre, pasa a unos registros c. $10 \%$ en Vallès 2 y menor en el resto de grupos. El caso de las incisiones/acanalados es más específico. Tiene un peso residual en Penedès 2, Vallès 2 y Litoral central y gana importancia en Vidre y es una técnica principal en el grupo Segre-Pirineos. El boquique y el sillon d'impressions sólo tienen presencia en Litoral central (en Sant Pau del Camp), en Segre-Pirineos, en Font Major y en Vidre.

Entre las combinaciones de matrices de la Fase 2, el cardial sobre cordón es numéricamente representativo en Penedès 2, Litoral central, Font Major (10-15\%) y a más distancia en la Draga.
En el tipo impresión simple sobre cordón, sólo Segre-Pirineos y Vidre tienen porcentajes superiores al $10 \%$. Por su parte, la combinación cardial con impresión o incisión es relevante en los grupos Penedès 2, Vallès 2, Vidre y Draga. En el resto de yacimientos su valor porcentual es poco importante. Por su parte, los vasos con impresión simple e incisión únicamente tienen presencia en Penedès 2, Litoral central, Vidre y sobretodo en Segre-Pirineos, si bien con porcentajes cercanos o menores al 5\%. Por último, la combinación de boquique con impresiones (simples o cardiales, sólo en Parco) sólo está presente en el grupo Segre-Pirineos, en una baja representación.

\subsubsection{Técnicas impresas detalladas}

Para aumentar la resolución de los datos decorativos, se han analizado las matrices impresas de manera pormenorizada. Las decoraciones incisas/acanaladas y los cordones lisos no han sido tenidos en cuenta. Ello reduce el número de vasos decorados a 597 efectivos. Este hecho ha agravado el problema de el Cavet, que ahora sólo cuenta con 10 efectivos. Por ese motivo, sus datos deben ser tomados, nuevamente, con prudencia. 


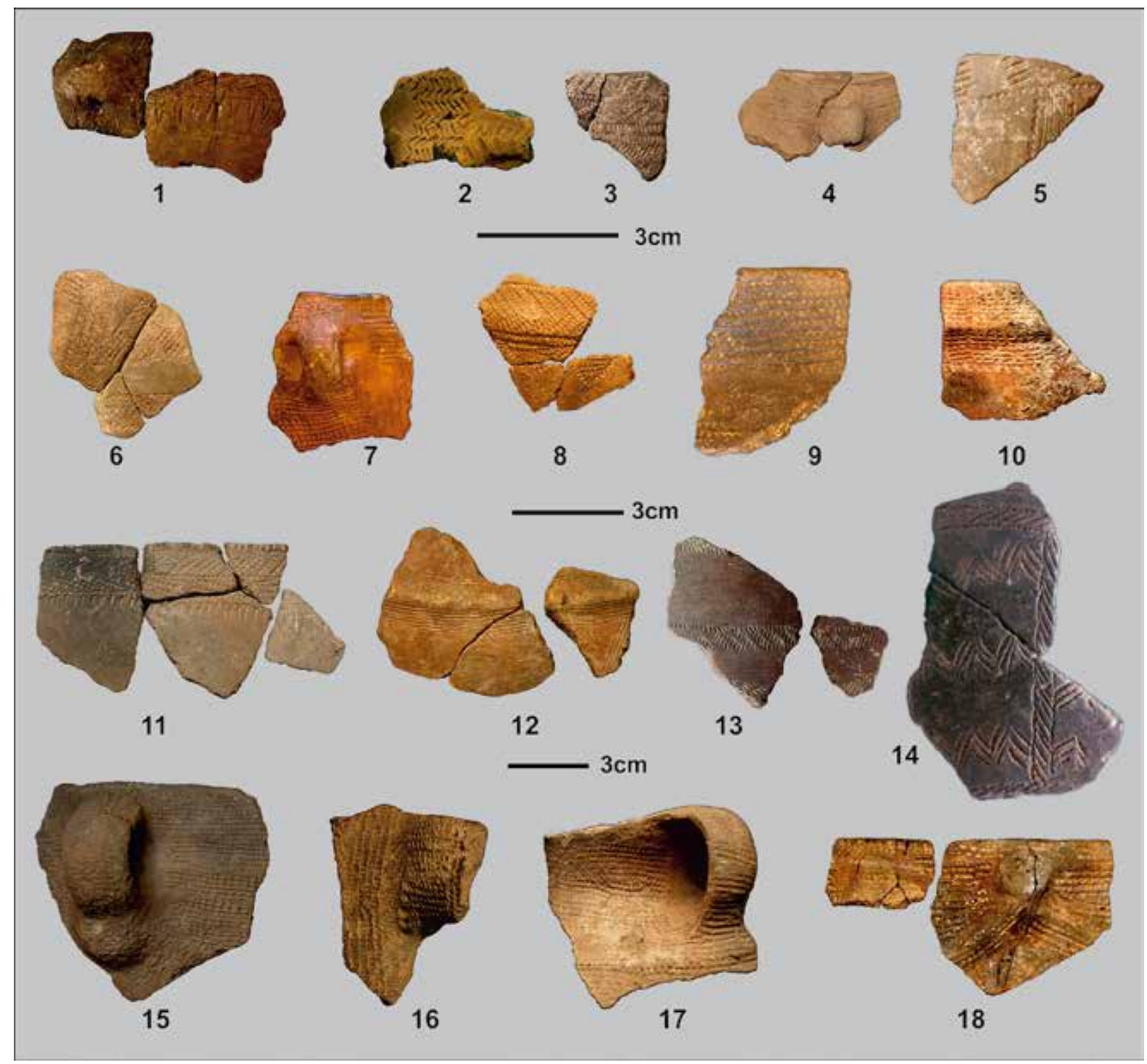

Fig. 5. Ejemplos de cerámicas decoradas de la Fase 2 (territorios litorales y prelitorales): Balma de l'Espluga $(1,2)$, Cova Bonica $(3,13,16)$, Cova de la Font Major (4,18). Cova del Bolet (5,7), Cova del Frare (6,11). Turó de Can Bellsolà (8). Sant Pau del Camp (9). Cova de la Guineu (10,17), Esquerda de les Roques del Pany $(12,15)$ y Cova del Vidre (14) / Decorated potteries from the Phase 2 (coastal and precoastal territories): Balma de l'Espluga $(1,2)$, Cova Bonica $(3,13,16)$, Cova de la Font Major $(4,18)$, Cova del Bolet $(5,7)$, Cova del Frare $(6,11)$, Turó de Can Bellsolà $(8)$, Sant Pau del Camp $(9)$, Cova de la Guineu $(10,17)$, Esquerda de les Roques del Pany $(12,15)$ and Cova del Vidre (14).

En la Fase 1, la impresión cardial oblicua como única matriz es la decoración mayoritaria, aunque los porcentajes varían mucho entre los tres territorios analizados. Es destacable observar que sólo en Penedès 1 se documenta la presencia de cardial arrastrado. Por contra, el cardial perpendicular es más habitual en Vallès 1 (y porcentualmente) en el Cavet. Las impresiones de instrumento tienen poco peso en la muestra, y el peine sólo está presente en un vaso de la Cova del Toll. Por su parte, las combinaciones entre matrices también son escasas. Mientras en el Cavet sólo se documentan en un vaso, en Penedès 1 sólo el cardial arrastrado con oblicuo y el oblicuo con perpendicu- lar tienen cierta presencia. En Vallès 1 esta dinámica cambia ligeramente. Vuelve a no documentarse la presencia de cardial arrastrado en ninguna combinación, que en cambio están presididas por el usos de cardial oblicuo con perpendicular y sobretodo del tipo oblicuo con nátex, además de la presencia -aunque escasa- de la asociación entre cardial oblicuo e incisión. En el yacimiento de la Cova de Can Sadurní c18, no analizado por nosotros, se documenta también la combinación cardial oblicuo con nátex durante la Fase 1 (Blasco et al., 2009).

En la Fase 2 el escenario cambia sustancialmente. El uso exclusivo de cualquier matriz impresa descien- 


\begin{tabular}{|c|c|c|c|c|c|c|c|c|c|c|c|c|c|c|c|c|c|c|}
\hline & A & B & C & D & E & $\mathbf{F}$ & $\mathbf{G}$ & $\mathbf{H}$ & I & J & $\mathbf{K}$ & $\mathbf{L}$ & M & N & 0 & $\mathbf{P}$ & $\mathbf{Q}$ & $\mathbf{R}$ \\
\hline \multicolumn{19}{|c|}{ Datos totales } \\
\hline Cavet & 5 & 0 & 1 & 0 & 1 & 0 & 0 & 0 & 0 & 0 & 0 & 0 & 2 & 0 & 1 & 0 & 0 & 10 \\
\hline Penedès 1 & 44 & 7 & 2 & 1 & 10 & 5 & 0 & 1 & 0 & 1 & 0 & 0 & 2 & 0 & 0 & 0 & 0 & 73 \\
\hline Vallès 1 & 19 & 0 & 7 & 1 & 0 & 6 & 0 & 0 & 1 & 0 & 8 & 3 & 4 & 0 & 0 & 0 & 0 & 49 \\
\hline Penedès 2 & 13 & 8 & 3 & 0 & 9 & 5 & 3 & 7 & 6 & 1 & 0 & 1 & 5 & 2 & 0 & 0 & 0 & 63 \\
\hline Vallès 2 & 20 & 2 & 5 & 0 & 0 & 1 & 0 & 2 & 5 & 2 & 2 & 8 & 5 & 1 & 0 & 0 & 0 & 53 \\
\hline Litoral central & 36 & 7 & 5 & 1 & 4 & 3 & 0 & 1 & 2 & 2 & 4 & 2 & 5 & 0 & 3 & 0 & 0 & 75 \\
\hline Vidre & 11 & 2 & 0 & 0 & 1 & 1 & 0 & 0 & 0 & 5 & 0 & 13 & 17 & 19 & 2 & 0 & 0 & 71 \\
\hline Font Major & 7 & 18 & 4 & 0 & 1 & 0 & 0 & 0 & 4 & 4 & 2 & 0 & 16 & 3 & 2 & 0 & 0 & 61 \\
\hline Draga & 10 & 0 & 5 & 0 & 0 & 2 & 0 & 20 & 3 & 2 & 0 & 2 & 2 & 1 & 0 & 0 & 0 & 47 \\
\hline Segre-Pirineos & 1 & 3 & 3 & 1 & 0 & 0 & 0 & 0 & 3 & 5 & 0 & 2 & 56 & 4 & 9 & 4 & 4 & 95 \\
\hline Total & 155 & 39 & 35 & 4 & 34 & 18 & 2 & 29 & 20 & 21 & 14 & 31 & 110 & 30 & 17 & 4 & 4 & 597 \\
\hline \multicolumn{19}{|c|}{ Datos porcentuales } \\
\hline Cavet & 50 & 0 & 10 & 0 & 10 & 0 & 0 & 0 & 0 & 0 & 0 & 0 & 20 & 0 & 10 & 0 & 0 & 100 \\
\hline Penedès 1 & 60,2 & 9,5 & 2,7 & 1,3 & 13,7 & 6,8 & 0 & 1,3 & 0 & 1,3 & 0 & 0 & 2,7 & 0 & 0 & 0 & 0 & 100 \\
\hline Vallès 1 & 38,7 & 0 & 14,2 & 2 & 0 & 12,2 & 0 & 0 & 2 & 0 & 16,3 & 6,1 & 8,1 & 0 & 0 & 0 & 0 & 100 \\
\hline Penedès 2 & 20,6 & 12,7 & 4,7 & 0 & 14,3 & 7,9 & 4,7 & 11,1 & 9,5 & 1,6 & 0 & 1,6 & 7,9 & 3,2 & 0 & 0 & 0 & 100 \\
\hline Vallès 2 & 39,2 & 3,9 & 9,8 & 0 & 0 & 1,9 & 0 & 3,9 & 9,8 & 3,9 & 1,3 & 15,6 & 9,8 & 1,9 & 0 & 0 & 0 & 100 \\
\hline Litoral central & 48 & 9,3 & 6,6 & 1,3 & 5,3 & 4 & 0 & 1,3 & 2,6 & 2,6 & 5,3 & 2,6 & 6,6 & 0 & 4 & 0 & 0 & 100 \\
\hline Vidre & 15,5 & 2,8 & 0 & 0 & 1,4 & 1,4 & 0 & 0 & 0 & 7 & 0 & 18,3 & 23,9 & 26,7 & 2,8 & 0 & 0 & 100 \\
\hline Font Major & 11,4 & 29,5 & 6,5 & 0 & 1,6 & 0 & 0 & 0 & 6,5 & 6,5 & 3,2 & 0 & 26,2 & 4,9 & 3,2 & 0 & 0 & 100 \\
\hline Draga & 21,2 & 0 & 10,6 & 0 & 0 & 4,2 & 0 & 42,5 & 6,3 & 4,2 & 0 & 4,2 & 4,2 & 2,1 & 0 & 0 & 0 & 100 \\
\hline Segre-Pirineos & 1,1 & 3,1 & 3,1 & 1,1 & 0 & 0 & 0 & 0 & 3,1 & 5,2 & 0 & 2,1 & 58,9 & 4,2 & 9,4 & 4,2 & 4,2 & 100 \\
\hline
\end{tabular}

Tabla 4: Tipos de matrices decorativas detalladas. A: Cardial oblicuo; B: Cardial arrastrado; C: Cardial perpendicular; D: Otra tipo de concha (no dentada); E: Cardial oblicuo+arrastrado; F: Cardial oblicuo+perpendicular; G: Cardial arrastrado+perpendicular; H: Cardial+peine; I: Peine; J: Cardial+impresión; K: Cardial+nátex cardial; L: Cardial+incisión; M: Impresión simple; N: Impresión+incisión; O: Boquique/Sillon; P: Boquique+cardial; Q: Boquique+impresión; R: Total / Detailed decorative techniques. A: Obliquous Cardial; B:Dragged Cardial; C: Perpendicular Cardial; D: Other shell impression (toothless): E: Oblicuous+Dragged Cardial; F: Oblicuous+perpendicular Cardial; G: Dragged+perpendicular Cardial; H: Cardial+Comb; I: Comb; J: Cardial+other impressed technique; K: Cardial+Umbo impression; L: Cardial+Inicision; M: Impression; N: Impression+incision; O: Boquique/Sillon; P: Boquique+Cardial; Q: Boquique+impressions; R: Total.

de de manera notoria, solamente en Litoral central, el uso de cardial oblicuo se mantiene cercano al 50\%. El cardial arrastrado pasa a ser una matriz preferente en Penedès 2 y sobretodo en Font Major, mientras que en Vallès 2 y Vidre no llega al 5\% y en Draga no se documenta. El tipo perpendicular sólo tiene una presencia cercana al $10 \%$ en Draga y Vallès 2. El uso de peine como matriz única tiene porcentajes entre 5-10\% sólo en los territorios de Penedès 2, Vallès 2, Draga y Font Major. Las impresiones simples son ampliamente las matrices predominantes en el territorio Segre-Pirineos y tienen mucha importancia en Font Major y Vidre (c.25\%).

Cuando nos centramos en las combinaciones de matrices, los tipos cardial oblicuo con arrastrado tiene un peso muy importante en Penedès 2 , pero está casi ausente en el resto de territorios. Otras combinaciones entre matrices cardiales no presentan cantidades representativas. La presencia de la asociación entre peine y cualquier tipo de impresión cardial tiene c. 15\% en Penedès 2 y más de un 40\% en la Draga. La impresión simple combinada con alguna técnica cardial tiene una importancia poco relevante en todos los territorios. En cambio, la asociación entre incisión y cardial posee una buena presencia (c.15-20\%) en Vallès 2 y en Vidre. La presencia de la combinación de cualquier tipo de cardial con la impresión del nátex de la concha tiene en la Fase 2 poco peso específico, siendo el territorio Litoral central el que más efectivos recuenta $(N R=4)$. El yacimiento de la Cova d'en Pau (nivel II), no analizado por nosotros y probablemente integrado en la Fase 2, también cuenta con un efectivo donde se combina el cardial oblicuo con el nátex (Tarrús, 1981).

Otras técnicas a tener en cuenta por su especial significación (a pesar de su baja frecuencia) son el boquique y el sillon d'impressions. El primero sólo se documenta en un vaso de el Cavet en la Fase 1 y también en Vidre, Font Major y Segre-Pirineos (en esta caso también combinado con impresiones simples y cardiales) durante la Fase 2. El sillon d'impressions, por su parte, sólo se ha documentado en Sant Pau del Camp (3 fragmentos de 3 vasos distintos en otros tantos silos), en la Fase 2. 


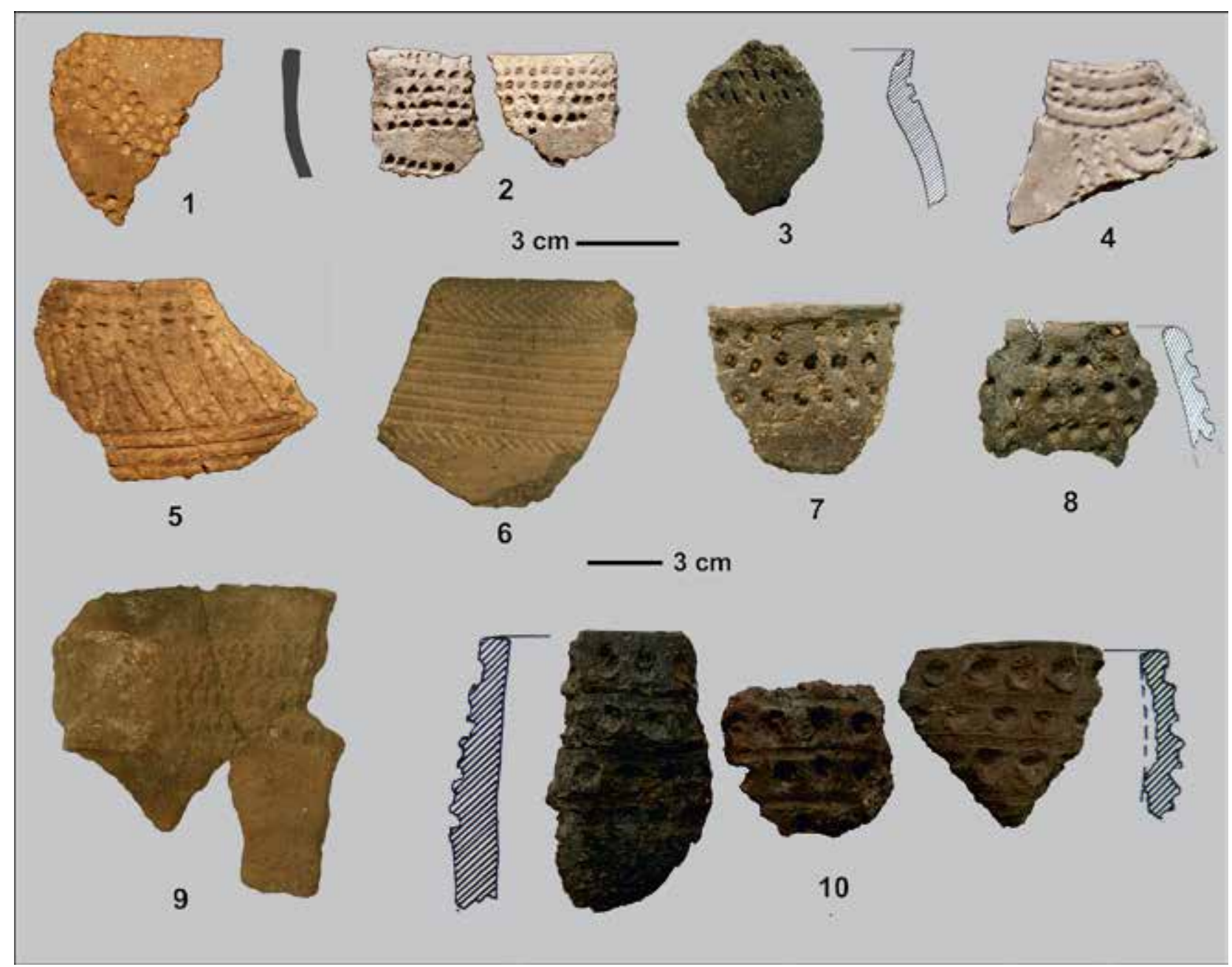

Fig. 6. Ejemplos de cerámicas decoradas de la Fase 2 (Segre-Pirineos): Cova Colomera $(1,2,4,5)$, Balma Margineda $(3,7,8,10)$ y Cova del Parco $(6,9)$ / Decorated potteries from the Phase 2 (Segre-Pyrenees region): Cova Colomera $(1,2,4,5)$, Balma Margineda $(3,7,8,10)$ y Cova del Parco $(6,9)$.

\subsubsection{Los motivos decorativos}

Como en casos anteriores, la fragmentación del registro ha impedido poder analizar la totalidad de los efectivos, por ese motivo la muestra se basa aquí en 646 vasos. Así, se han determinado un total de 461 motivos sencillos y 115 complejos. Además, es habitual que en un vaso se dé más de un solo motivo. En este caso los denominamos combinados, y hemos documentado un total de 70 .

Este análisis demuestra que los motivos más sencillos son más numerosos en los yacimientos de los grupos de la Fase 1 así como en los territorios Segre-Pirineos, Vidre y Font Major. En estos predominan las franjas horizontales simples (A1) y las limitadas (A2), a veces combinadas con franjas descendientes (simples D1 y limitadas D2), los cordones lisos únicos (I1) y los cordones con impresiones simples (14). Otros tipos como las franjas anchas (E1 y E2) y los cordones paralelos (I2) tienen menor presencia.
En cambio, los motivos de tipo complejo son más habituales de los grupos Penedès 2, Vallès 2 y Litoral central, todos ellos de la Fase 2 y situados en la geografía de tradición cardial. Los tipos combinados tienen un comportamiento errático pero por norma general son más habituales en los grupos de la Fase 2. En este caso tienen una presencia estable las franjas horizontales múltiples simples (B1) y limitadas (B2), también en las que de estas franjas descienden otras verticales (C1 y C2). Las franjas anchas con cordón (E3), las metopas (G1), todos aquellos tipos con franjas ascendentes o descendentes oblicuas $(H)$ y los meandros (D3-6) tienen una representación más esporádica.

Estos datos no sólo diferencian más los territorios considerados cardiales entre una fase y otra, sino que durante la Fase 2, los yacimientos del territorio Segre-Pirineos, Vidre y Font Major tienen muy poca relación con los de Penedès 2, Vallès 2, Draga y Litoral central. 


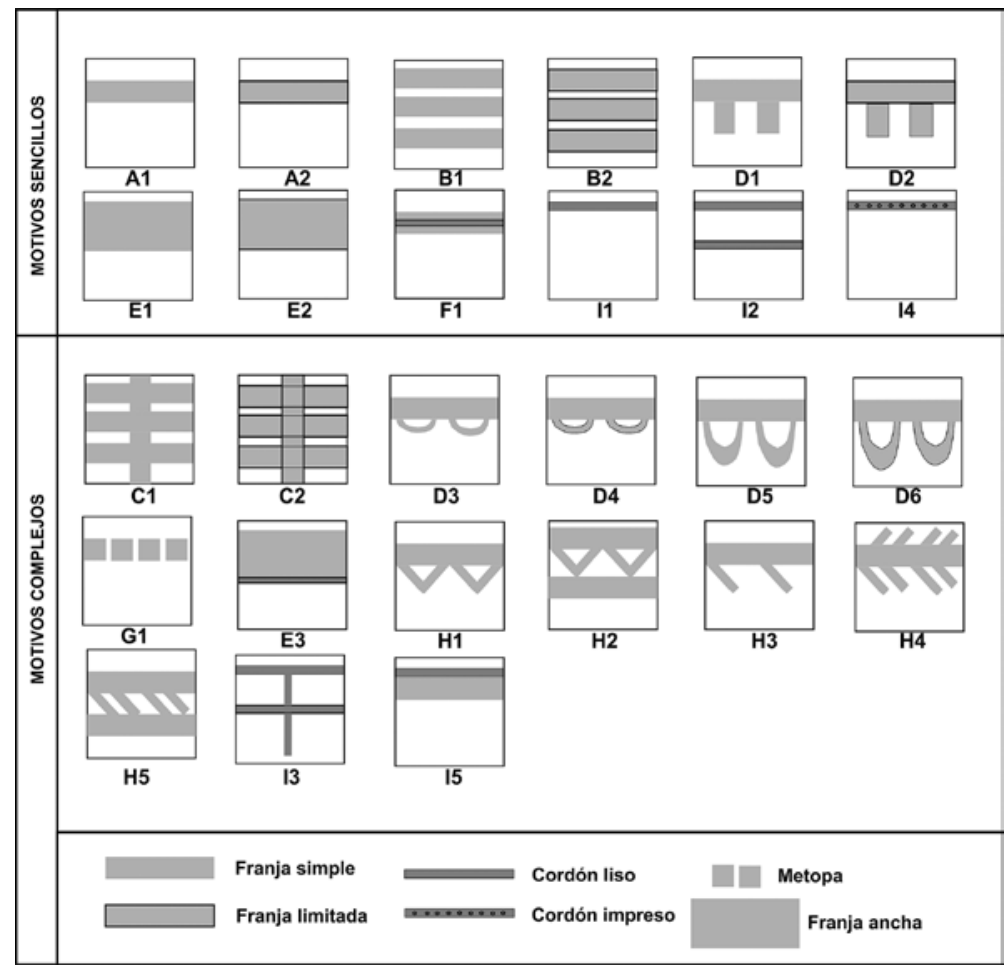

Fig. 7. Motivos documentados en los diferentes conjuntos cerámicos analizados / Decorative motifs in the different pottery groups.

\begin{tabular}{|c|c|c|c|c|c|c|c|c|c|c|c|c|c|c|c|c|c|c|c|}
\hline & $\frac{\mathfrak{z}}{4}$ & กั & $\underset{\mathbf{u}}{\mathbf{z}}$ & $\overline{4}$ & $=$ & $\simeq$ & \pm & 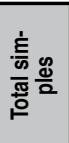 & నิ & ปั & 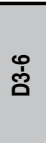 & E & జึ & $\stackrel{\text { 오 }}{\frac{1}{x}}$ & $\underline{m}$ & 토 & 퐁 $\frac{\mathscr{0}}{\frac{0}{0}}$ & 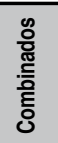 & 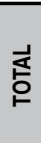 \\
\hline \multicolumn{19}{|c|}{ Datos totales } & \\
\hline Cavet & 8 & 1 & 0 & 1 & 3 & 2 & 0 & 15 & 0 & 0 & 0 & 0 & 0 & 0 & 0 & 0 & 0 & 0 & 15 \\
\hline Penedès 1 & 27 & 4 & 4 & 0 & 17 & 0 & 2 & 54 & 2 & 0 & 2 & 1 & 0 & 4 & 1 & 0 & 10 & 7 & 71 \\
\hline Vallès 1 & 16 & 4 & 3 & 2 & 9 & 0 & 1 & 35 & 2 & 2 & 1 & 0 & 0 & 1 & 1 & 0 & 7 & 5 & 49 \\
\hline Penedès 2 & 6 & 1 & 3 & 0 & 8 & 2 & 1 & 21 & 10 & 1 & 1 & 0 & 0 & 1 & 1 & 1 & 15 & 11 & 47 \\
\hline Vallès 2 & 20 & 3 & 0 & 1 & 14 & 2 & 4 & 44 & 4 & 3 & 2 & 1 & 1 & 3 & 3 & 0 & 17 & 5 & 66 \\
\hline Litoral central & 25 & 2 & 0 & 5 & 14 & 1 & 3 & 50 & 6 & 2 & 3 & 1 & 2 & 3 & 2 & 1 & 20 & 8 & 78 \\
\hline Vidre & 47 & 6 & 0 & 0 & 12 & 2 & 4 & 71 & 4 & 1 & 0 & 4 & 0 & 0 & 2 & 0 & 11 & 13 & 95 \\
\hline Font Major & 19 & 7 & 1 & 9 & 13 & 3 & 0 & 52 & 3 & 1 & 1 & 2 & 2 & 1 & 3 & 2 & 15 & 4 & 71 \\
\hline Draga & 10 & 7 & 1 & 3 & 8 & 0 & 0 & 29 & 2 & 2 & 3 & 1 & 1 & 0 & 2 & 0 & 11 & 11 & 51 \\
\hline Segre-Pirineos & 45 & 5 & 6 & 2 & 13 & 4 & 15 & 90 & 4 & 0 & 2 & 2 & 0 & 0 & 1 & 0 & 9 & 6 & 105 \\
\hline Total & 233 & 40 & 18 & 23 & 111 & 16 & 30 & 471 & 35 & 10 & 13 & 10 & 4 & 13 & 16 & 4 & 105 & 70 & 648 \\
\hline \multicolumn{19}{|c|}{ Datos porcentuales } & \\
\hline Cavet & 53,3 & 6,6 & 0 & 6,6 & 20 & 12,2 & 0 & 100 & 0 & 0 & 0 & 0 & 0 & 0 & 0 & 0 & 0 & 0 & 100 \\
\hline Penedès 1 & 38 & 5,6 & 5,6 & 0 & 23,9 & 0 & 2,8 & 75,9 & 2,8 & 0 & 2,8 & 1,4 & 0 & 5,6 & 1,4 & 0 & 11,2 & 9,8 & 100 \\
\hline Vallès 1 & 34 & 8,4 & 6,3 & 4,2 & 19,1 & 0 & 2,1 & 78,2 & 4,2 & 4,2 & 2,1 & 0 & 0 & 2,1 & 2,1 & 0 & 10,6 & 10,6 & 100 \\
\hline Penedès 2 & 12,7 & 2,1 & 6,3 & 0 & 17 & 4,2 & 2,1 & 65,9 & 21,2 & 2,1 & 2,1 & 0 & 0 & 2,1 & 2,1 & 2,1 & 10,6 & 23,4 & 100 \\
\hline Vallès 2 & 30,3 & 4,5 & 0 & 1,5 & 21,2 & 3 & 6 & 66,5 & 6 & 4,5 & 3 & 1,5 & 1,5 & 4,5 & 4,5 & 0 & 25,5 & 7,5 & 100 \\
\hline Litoral central & 32 & 2,5 & 0 & 6,4 & 17,9 & 1,2 & 3,8 & 63,8 & 7,6 & 2,5 & 3,8 & 1,2 & 2,5 & 3,8 & 2,5 & 1,2 & 25,3 & 11,1 & 100 \\
\hline Vidre & 55,2 & 7 & 0 & 0 & 14,1 & 2,3 & 4,7 & 76,4 & 4,7 & 1,1 & 0 & 4,7 & 0 & 0 & 2,3 & 0 & 8,2 & 15,2 & 100 \\
\hline Font Major & 26,7 & 9,8 & 1,4 & 12,6 & 18,3 & 4,2 & 0 & 77,4 & 4,2 & 1,4 & 1,4 & 2,8 & 2,8 & 1,4 & 4,2 & 2,8 & 16,9 & 5,6 & 100 \\
\hline Draga & 19,6 & 13,7 & 1,9 & 5,8 & 0 & 0 & 0 & 60,7 & 3,9 & 3,8 & 5,8 & 1,9 & 1,9 & 0 & 3,8 & 0 & 17,6 & 21,5 & 100 \\
\hline Segre-Pirineos & 42,8 & 4,7 & 5,7 & 1,9 & 12,3 & 3,8 & 14,2 & 89,5 & 3,8 & 0 & 1,9 & 1,9 & 0 & 0 & 0,9 & 0 & 8,5 & 5,7 & 100 \\
\hline
\end{tabular}

Tabla 5: Motivos decorativos documentados en la muestra / Decorative motifs in the pottery sample. 


\begin{tabular}{|l|l|l|l|l|}
\hline & Simples & Complejos & Combinados & Total \\
\hline Cavet & $100(15)$ & 0 & 0 & $100(15)$ \\
\hline Penedès 1 & $76(54)$ & $14,1(10)$ & $9,8(7)$ & $100(71)$ \\
\hline Vallès 1 & $74,4(35)$ & $14,9(7)$ & $10,6(5)$ & $100(47)$ \\
\hline Penedès 2 & $44,6(21)$ & $31,9(15)$ & $23,4(11)$ & $100(47)$ \\
\hline Vallès 2 & $66,6(44)$ & $25,7(17)$ & $7,5(5)$ & $100(66)$ \\
\hline Litoral central & $64,1(50)$ & $25,6(20)$ & $10,2(8)$ & $100(78)$ \\
\hline Vidre & $74,7(71)$ & $11,5(11)$ & $13,6(13)$ & $100(95)$ \\
\hline Font Major & $73,2(52)$ & $21,1(15)$ & $5,6(4)$ & $100(71)$ \\
\hline Draga & $56,8(29)$ & $21,5(11)$ & $21,5(11)$ & $100(51)$ \\
\hline Segre-Pirineos & $85,7(90)$ & $8,5(9)$ & $5,7(6)$ & $100(105)$ \\
\hline Total & 461 & 115 & 70 & 646 \\
\hline
\end{tabular}

Tabla 6: Síntesis de los motivos decorativos, según su tipo (en paréntesis el número de casos) / Synthesis of decorative motifs by type (in parentheses the number of cases).

\subsection{Síntesis de resultados}

La Fase 0, caracterizada en base a las fechas de Guixeres A y de Cavet 2014, cuenta con pocos vestigios. En el Cavet, el material vinculado con esa datación no permite realizar ninguna aproximación, otros todavía no datados si y por tanto su valoración queda aparcada. En cambio, en Les Guixeres de Vilobí se ha detectado un lote de materiales cerámicos que pueden ser vinculados con la datación más antigua del registro. Este registro se sitúa en la base de la secuencia y en estos momentos está en proceso de estudio. Se trata de un mínimo de 4 vasos que difieren totalmente de los registros catalanes vinculados con el cardial franco-ibérico (Martins et al., 2015).

Sobre de las técnicas decorativas, se ha observado que durante la Fase 1 hay una baja cantidad de matrices decorativas y con pocas combinaciones. Este hecho es completamente opuesto en los territorios de la Fase 2: Tarragona interior, Ebro, Penedès 2, Vallès 2, Litoral central y Girona interior. En estas zonas se da un mayor número de matrices utilizadas. Las técnicas cardiales están presentes de manera mayoritaria en los territorios de la Fase 1 y 2 en los territorios litorales y prelitorales, mientras que en el interior (Vidre, Segre-Pirineos) éstas tienen menor importancia en favor de las impresiones simples. Por su parte, los cordones lisos son normalmente la segunda técnica más utilizada en la decoración de los vasos, en todos los territorios y fases por los que trasciende como un elemento de fondo y generalizado. Las combinaciones, limitadas casi al cardial sobre cordón en la Fase 1, tienen mayor presencia en Vallès 1 que en Penedès 1 y Cavet. En la Fase 2, las características decorativas se polarizan más y la percepción de territorialidad aumenta. Disminuye en los tramos de tradición cardial el uso del cardial como única matriz (Vallès 2, Penedès 2, Litoral central, Girona interior), mientras que tiene un peso significativo en Tarragona interior. En cambio, su importancia es relativa en Ebro y pasa a ser una técnica totalmente secundaria en Se-
gre-Pirineos. Los cordones lisos se mantienen estables y las combinaciones, en cualquiera de sus variantes, ganan mucho peso.

Después de este repaso general a las características de las producciones por fases, vamos a detallar la existencia de las especificidades cronológicas y geográficas más importantes:

a) Cardial arrastrado. Este tipo de impresión aparece ya desde la Fase 1 en la región Penedès 1 (y en Tarragona litoral con escasa presencia). En la Fase 2, se mantiene en Penedès 2 y se extiende con mucha fuerza en la zona Tarragona interior. Está también presente, aunque con menor entidad, en Vallès 2, en Litoral central y en el Ebro. Esta técnica se documenta en otros territorios cardiales: en el sur de Francia de manera muy escasa (Baume d'Oullins -Roudil \& VAN Willigen, 2002y en Leucate-Corrège, Guilaine et al., 1984) y también en algunas localizaciones valencianas como Cova de I'Or y Cova de les Cendres (Bernabéu, 1989; Garcia Borja et al., 2011). La localización cronológica de esta técnica es confusa, sin embargo en los casos de Or VI y Cendres X su presencia es esporádica en las fases más antiguas (Bernabéu, 1989: 115). En Aragón no está documentado su uso (Ramón, 2006).

b) Nátex cardial. Esta característica impresión cardial tiene una localización muy parcial en el territorio analizado. Durante la Fase 1 se documenta sólo en Vallès 1 (Toll), mientras que durante la Fase 2 se mantiene en Vallès 2 y se extiende su uso hacia el Litoral central. Fuera de nuestro estudio, el nátex también está documentado en la Cova de Can Sadurní c18 (Blasco et al., 2009) durante la Fase 1 y en la Cova d'en Pau (Tarrús, 1981) y en la Cova Gran y Cova Freda de Montserrat (Baldellou et al., 1989), probablemente asociado a conjuntos de la Fase 2. En Francia sólo está documentado en la Grotte de Pont fuera de contexto (Guilaine et al., 2008), mientras que en Aragón es inexistente. Por su parte, en Valencia es un recurso muy habitual documentado en todos los yacimientos mejor conocidos (Bernabéu, 1989; Bernabéu et al., 2011; García Borja et al., 2012). Parece distribuirse a lo largo de todas las secuencias, pero con un peso mayor al inicio del Neolítico Cardial (Bernabéu, 1989).

c) Cardial perpendicular anárquico. Si bien este tipo de impresión se da en muchos yacimientos de todos los periodos, no tiene una importancia latente y su presencia no es nunca de primer orden, sino puntual o complementaria. Sin embargo, en dos yacimientos de la Fase 0 o Fase 1, Cavet y Guixeres de Vilobí, encontramos unos pocos efectivos donde se dan estas impresiones de concha, perpendiculares y en disposición anárquica y vertical. Este tipo de impresión está bien presente en diferentes horizontes neolíticos precardiales (Binder \& Maggi, 2001; Manen, 2007; Bernabéu et al., 2009). Relacionado con horizontes cardiales, algunos autores sugieren que pueda tratarse de un rasgo arcaizante (García 


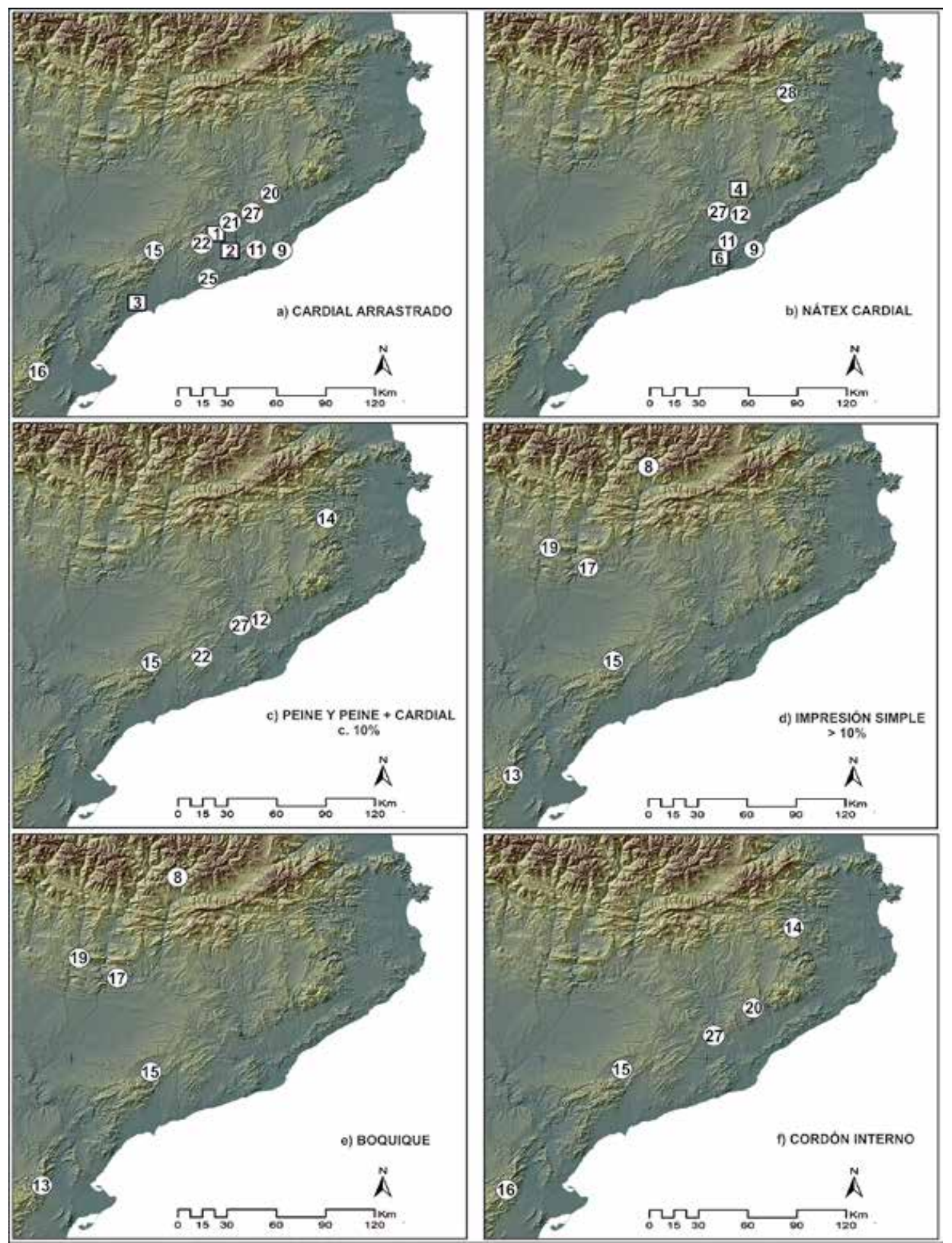

Fig. 8. Elementos decorativos característicos repartidos en el territorio según la fase cronológica (cuadrado=Fase 1; redonda=Fase 2). 1 Guixeres de Vilobí; 2 La Serreta; 3 Cavet; 4 Cova del Toll; 5 Can Roqueta II; 6 Cova de Can Sadurní; 7 Balma del Serrat del Pont; 8 Balma Margineda; 9 Sant Pau del Camp; 10 Plaça Vila de Madrid; 11 Cova Bonica; 12 Cova del Frare; 13 Turó de Can Bellsolà; 14 La Draga; 15 Cova de la Font Major; 16 Cova del Vidre; 17 Cova del Parco; 18 Cova Gran de Santa Linya; 19 Cova Colomera; 20 Balma de l'Espluga; 21 Cova de la Guineu y Cova del Bolet; 22 Esquerda de les Roques del Pany; 23 Coll Blanc; 24 Cova de Sant Llorenç; 25 Cova Foradada; 26 Plansallosa; 27 Cova Gran i Cova Freda; 28 Cova d'en Pau; 29 Cova de l'Arbreda; 30 Font del Ros (Autor base cartográfica: Gerard Remolins) / Characteristic decorative elements spread over the territory according to the chronological phase (Phase $1=$ square, round = Phase 2). 1 Guixeres de Vilobí; 2 La Serreta; 3 Cavet; 4 Cova del Toll; 5 Can Roqueta II; 6 Cova de Can Sadurní; 7 Balma del Serrat del Pont; 8 Balma Margineda; 9 Sant Pau del Camp; 10 Plaça Vila de Madrid; 11 Cova Bonica; 12 Cova del Frare; 13 Turó de Can Bellsolà; 14 La Draga; 15 Cova de la Font Major; 16 Cova del Vidre; 17 Cova del Parco; 18 Cova Gran de Santa Linya; 19 Cova Colomera; 20 Balma de I'Espluga; 21 Cova de la Guineu y Cova del Bolet; 22 Esquerda de les Roques del Pany; 23 Coll Blanc; 24 Cova de Sant Llorenç; 25 Cova Foradada; 26 Plansallosa; 27 Cova Gran i Cova Freda; 28 Cova d'en Pau; 29 Cova de l'Arbreda; 30 Font del Ros (Base map: Gerard Remolins). 
Atiénzar, 2010) a partir de registros sin contexto como la Cova Ampla de Montgó. Sin embargo, en otras secuencias esta técnica se encuentra en un Cardial pleno e incluso reciente (García Borja et al., 2011).

d) Cordones internos. Este tipo de cordón, asociado siempre a otro externo a la misma altura y con morfología cercana a la cresta, tiene una distribución muy limitada en el NE peninsular y siempre en la Fase 2. Se documenta en Girona (Draga), en Vallès 2 (l'Espluga), Penedès 2 (Pany), en Tarragona interior (Font Major) y en Ebro (Vidre). En estos yacimientos, su presencia es casi testimonial (1-3 vasos). Fuera de nuestro análisis, estos cordones también se documentan en la Cova Freda de Montserrat, Cova d'en Pau y Reclau Viver (Tarrús \& Bosch, 1990). Por su parte, no se conocen paralelos en Valencia y está escasamente documentado en el Languedoc occidental. En cambio, sí está bien representado en la cuenca baja del Ródano en yacimientos como Baume Bourbon y Grotte de Taï entre otros (Guilaine et al., 2008). En estos casos se asocian a conjuntos de tradición Epicardial con dataciones cercanas a 6200-6150 BP (Manen et al., 2010).

e) Impresiones simples y peine. Las impresiones simples y complejas (que no se corresponden con ningún tipo de concha) documentadas en este trabajo tienen morfologías muy diversas atendiendo a múltiples herramientas: punzones circulares, medias cañas, uñadas y matrices complejas (peines). El uso de este último tipo empieza de una manera muy esporádica en la Fase 1 en Penedès y Vallès y se extiende, durante la Fase 2 por numerosos territorios vinculados a las zonas que podemos denominar de influencia Cardial (Penedès 2, Vallès 2, Litoral central, Girona interior). Por su parte, las impresiones simples sólo tienen presencia durante la Fase 2 fuera de estos territorios. Su máxima representación se da en Segre-Pirineos, pero también en Font Major (Tarragona interior) y Vidre (Ebro). En otros territorios, el uso de impresiones simples y peines también se documenta de manera escasa en las fases iniciales y proliferan de manera importante en estadios avanzados del Neolítico inicial (Manen, 2002; Bernabéu et al., 2011; García Borja et al., 2011).

f) Incisiones/Acanalados. Esta técnica tiene bajos porcentajes (o nulos) en la mayor parte de los yacimientos de territorios cardiales, tanto en la Fase 1 como en la 2. Sólo el caso de el Cavet es significativo durante las fases más antiguas, si bien cuenta con pocos efectivos. Durante la Fase 2 en zonas como Segre-Pirineos, cuenta con un mayor peso específico y llega a significar la tercera técnica más utilizada en asentamientos como la Cova Colomera. Esta técnica es poco recurrente en horizontes antiguos de otros territorios, y sólo gana presencia a partir de 6200 BP en horizontes cardiales muy evolucionados (Bernabéu et al., 2011) y sobretodo en contextos epicardiales (García Martínez de Lagrán et al., 2011; Manen et al., 2010). g) Boquique. Técnica decorativa documentada en territorios ajenos a los focos cardiales tradicionales, se encuentra siempre en bajos porcentajes (<10\%) y en asentamientos con dataciones de la Fase 2. Cova Colomera, Cova del Parco y Balma Margineda en Segre-Pirineos, la Cova de la Font Major en el interior de Tarragona y en la Cova del Vidre en el tramo final del Ebro son las localizaciones más destacadas. Solamente está presente en los territorios donde se reproduce un Epicardial antiguo y avanzado (Alday \& Moral, 2011). Sin embargo, tiene un recorrido cronológico extraordinariamente amplio en estas regiones (Alday, 2008) por lo que su importancia cronocultural es, cuanto menos, discutible. La Cueva dels Trocs es el enclave cercano con mayor cantidad de efectivos, aunque en ninguna de sus fases supera el 6\% de presencia (Rojo et al., 2013).

h) Sillon d'Impressions. Esta técnica se asocia a horizontes litorales precardiales (Manen, 2007; BERNABÉU et al., 2009). En el NE peninsular solamente ha sido documentado en Sant Pau del Camp. Los efectivos se asocian a silos con materiales claramente integrables dentro del Cardial franco-ibérico. Además, en uno de los casos, el fragmento se encuentra en un silo ya datado (s.2: $6250 \pm 40 B P)$, con un resultado más reciente de lo que sería esperable para un conjunto Impressa. Esto podría indicar una recurrencia técnica o quizá se trate de "ruido de fondo", un material abandonado en superficie e incorporado en otro conjunto (silo) a posteriori.

\section{DISCUSIÓN Y CONCLUSIONES}

La definición de las primeras fases neolíticas del extremo occidental mediterráneo dieron un vuelco con la publicación de los resultados de Portiragnes en el sur de Francia (Manen, 2002; Guilaine et al., 2007) y de El Barranquet de Oliva en el levante peninsular (Bernabéu et al., 2009). Ello amplió el escenario conocido hasta ese momento y reducido casi a los conjuntos de Arene Candide y Pendimoun entre otros (Binder et al., 1993; Binder \& Maggi, 2001). Estos resultados mostraban una realidad plenamente neolítica anterior al grupo Cardial. En el conjunto que hemos analizado, un pequeño lote de materiales de Les Guixeres de Vilobí sin paralelos en los registros catalanes, puede integrarse en este horizonte (Martins et al., 2015). Por su parte, los datos ya comentados de el Cavet (poca representatividad) y de Sant Pau del Camp (sillon d'impressions mezclado con elementos cardiales) no permiten mayores apreciaciones por el momento.

Después de una fase formativa con muy pocos vestigios en Cataluña, los materiales de la Fase 1 ya se sitúan dentro de los cánones del Cardial franco-ibérico estrictos. En esta primera gran ocupación neolítica, los espacios mejor representados son Penedès 1, Vallès 1 y Cavet. A estos hay que añadir los datos poco numerosos de la Balma del Serrat del Pont III.4 (Alcalde et al., 2002) y de la Balma Margineda c3b (Oms et al., en 2016b), así como de la Cova de Can Sadurní c18 (Edo 
et al., 2011), con una importante colección todavía en parte inédita. En este contexto se reproduce una intensa ocupación de los llanos litorales y sobretodo prelitoreles al norte y al sur del río Llobregat. Las ocupaciones de Serrat del Pont y Margineda no se deben corresponder con los grupos procedentes del eje del Llobregat sino de otras localizaciones del litoral meridional francés o de la costa gerundense. Desde esta fase se ha comprobado la relativa diversidad existente entre los conjuntos cerámicos del primer cardial del NE peninsular. Ello podría responder a diferentes comunidades con una base cultural común, pero ya con evoluciones independientes. Si esta manera de hacer se produjo antes de la elección de un territorio o después al estar asentados ya en él, es algo que no podemos dilucidar.

A partir de la Fase 2, la especificidad territorial aumenta y se ocupan nuevos espacios en aquellas zonas no frecuentadas durante la Fase 1. Se percibe una continuidad en los territorios de Penedès y Vallès y se suma a este espacio el litoral central con Sant Pau del Camp y Cova Bonica. Todos ellos muestran particularidades en los registros cerámicos decorados que les mantienen como entidades territorialmente definibles. El extremo nordeste se ve representado por la Draga y otros asentamientos no incluidos en este trabajo como la Cova d'en Pau y la Cova de l'Arbreda entre otros (Tarrús, 1981) y un poco más tarde la primera ocupación de Plansallosa (Bosch et al., 1998). En la Tarragona interior, la Cova de la Font Major representa un asentamiento donde se mezclan técnicas de clara tradición cardial como la técnica de arrastre (probablemente procedente del vecino Penedès), con otras técnicas decorativas, sobretodo las impresiones de instrumento e incluso el boquique. En el mismo territorio pero al final de la fase, Coll Blanc demuestra ya la preeminencia de las decoraciones impresas de instrumento y la nula incidencia de las técnicas cardiales (Bravo et al., 2014). Más al sur, esta mezcla es aún más evidente en la Cova del Vidre, que presenta mayores analogías con registros del Bajo Aragón (Utrilla \& Domingo, 2014; Rojo et al., 2015) que con otros de Cataluña. Pero donde se percibe una ruptura radical es al oeste del río Segre. Ahí se documentan numerosos yacimientos donde las impresiones cardiales son casi inexistentes o son anecdóticas enfrente de las impresiones de instrumento, incisiones/acanalados e incluso el boquique. La Cova del Parco, la Balma Margineda c3F, c3f y c3a y la Cova Colomera CV10, CE14 y CE13 son sus mejores ejemplos. Los registros de estos yacimientos, clasificables dentro de un Neolítico de cerámicas impresas y acanaladas, tienen claras analogías con los materiales de los yacimientos del interior languedociense (Oms et al., 2012), del Alto Aragón como la Cueva del Moro de Olvena (Ramón, 2006) y la Cueva dels Trocs (Rojo et al., 2013) e incluso con registros del valle del Ebro (Rojo et al., 2008).

Este fenómeno de polarización territorial parece reproducirse en otros importantes focos de ocupación neolítica pionera. En las regiones de la Provenza y Lan- guedoc en Francia, se ha detectado un patrón de ocupación similar. Importante presencia de asentamientos de una fase antigua en las zonas litorales en Centre Ville y Grotte de Cap Ragnon y rápidas incursiones hacia los territorios interiores al este y oeste del Ródano con ejemplos como la Balme d'Oullins, Baume de Ronze, Grotte de Fontbrégoua y Abri de Pendimoun (Van Willigen et al., 2009; Le Bras-Goude et al., 2010; Binder y Sénépart, 2010; Defranould, 2012), con dataciones c. 6500-6400 BP todos ellos sobre taxones de vida corta. Más al sur, sólo algunos materiales de los asentamientos litorales destruidos de Leucate-Corrège y les Dunes ofrecen datos sobre ocupaciones pioneras (Guilaine et al., 1984; Montjardin \& Cablat, 2004), mientras que al interior, los conjuntos de Grotte Gazel A, Abri de Jean Cros 2a-b y Grotte de Camprafaud c.19 son numéricamente anecdóticos (Guilaine et al., 1979; Manen, 2000) y no disponen de dataciones recientes.

Durante una hipotética Fase 2 en el sur de Francia (a partir de c.6300 BP), igual que sucede en Cataluña, la segmentación geográfica aumenta y ello se ve corroborado en ciertos aspectos de las producciones cerámicas. Con el Ródano como línea de contacto, al este se sitúan los asentamientos de tradición cardial, Baratin y Les Petites Bâties, mientras que al oeste se encuentran aquellos con unas producciones típicamente asimilables al Neolítico Epicardial antiguo (cerámicas impresas, acanaladas y con cordones ortogonales): Grotte de Taï, Balme Bourbon y Mas de Vignoles X (Manen et al., 2010). Más al sur, en el Languedoc, la mayor parte de asentamientos del interior se situarían dentro de un Neolítico de cerámicas impresas y acanaladas de tipo Epicardial, con los ejemplos de Grotte de Camprafaud c.18-16, Roc de Dourgne c.5 y Grotte Gazel B (Guilaine et al., 1993; Manen, 2000). Estos conjuntos difieren claramente de la mayor parte del registro de Leucate-Corrège (tardío, muy similar al de la Draga) e incluso de Aspre del Paradís (Manen et al., 2001), ambos de filiación Cardial indudable.

En Valencia, los diferentes grupos geográficos establecidos por Garcia Borja y otros (2012) ya se encuentran formados durante una primera fase cardial: en los territorios costeros la ya citada Cova de les Cendres y en el interior la Cova de l'Or, la Cova de la Sarsa, la Cova Fosca de la Vall d'Ebo y el Mas d'Is, y se ampliarían durante una hipotética fase reciente, con ejemplos como la Cova d'en Pardo (Soler et al., 2013) y Benámer (Torregrosa et al., 2011). Si bien no se conocen aún datos cerámicos específicos para los diferentes grupos propuestos ya que se dispone de visiones individuales (Bernabéu, 1989) o de conjunto (Bernabéu et al., 2011), se propone un funcionamiento similar al ya apuntado en Cataluña, donde existirían especificidades estilísticas a nivel territorial.

En todos los casos mencionados, la colonización Cardial del extremo mediterráneo occidental se muestra como un fenómeno homogéneo a escala temporal, pero no a escala cultural. Si bien los rasgos culturales y económicos son comunes, la variación territorial (a escala local o regional) es acusada. Esto sucede desde los primeros 


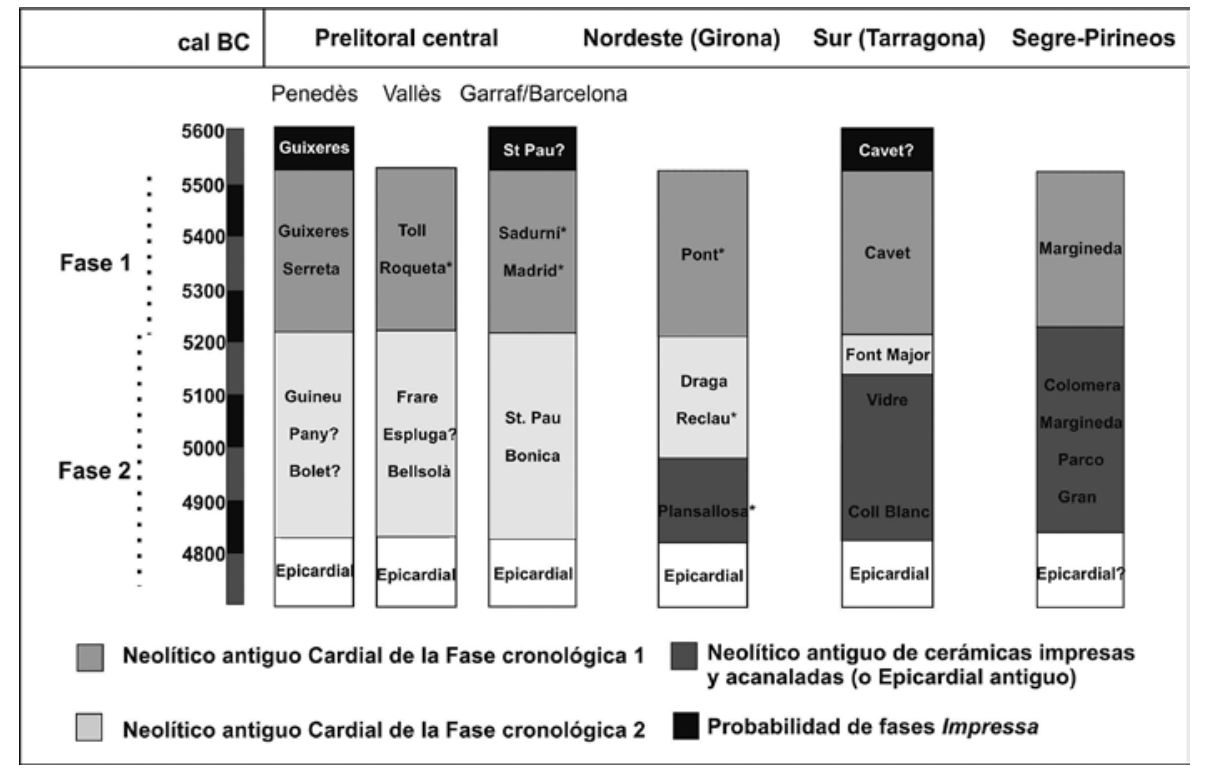

Fig. 9. Esquema cronológico y territorial del desarrollo del Neolítico inicial en el NE de la Península Ibérica / Chronological and territorial development scheme of the early Neolithic of NE of Iberia. momentos de esta expansión, y se ve ampliado durante una fase evolucionada del fenómeno Cardial. No podemos todavía conocer si estos mecanismos se corresponden con llegadas de diferentes grupos que ocupan biotopos diversos o, si por contra, responden a evoluciones internas durante escalas cronológicas muy breves. Tampoco es descartable un mecanismo de flujo-reflujo que afecte y relacione a diferentes territorios durante una misma fase cronológica. Durante la Fase 2, esta problemática se agudiza en el NE peninsular cuando entran en contacto en diferentes territorios el Neolítico Cardial y el de cerámicas impresas y acanaladas (Epicardial). Este "otro" Neolítico tiene, en general, pocas afinidades decorativas con el horizonte Cardial y se le presupone un origen diferente, que se desarrollaría por vía continental (Van Willigen, 2004), en un lapso radiocarbónico más tardío (en muestras de vida corta y doméstica) que la ocupación Cardial (García Martínez de Lagrán, 2014; Bernabéu \& Martí, 2014). En la Catalunya litoral y prelitoral, el Epicardial es siempre más reciente (Martín et al., 2010) y se alarga hasta un máximo de 4600 cal BC a partir de las dataciones disponibles (en muestras de vida corta) de Pou Nou, Cova del Frare (Martins et al., 2015) y de la Cova de Sant Llorenç (Borrell et al., 2014). Entonces, la visión homogénea de la colonización cardial debe ser matizada y completada en el futuro con trabajos y discusiones complementarias, que mejoren la contextualización entre registro cerámico y datación radiocarbónica (de calidad y representativa) y su relación con el Epicardial, tanto local como del interior. También será necesario avanzar en los análisis tecnológicos de las producciones cerámicas y analizar desde una visión parecida los usos del doble bisel, la morfología de las hoces líticas (Ibañez et al., 2015) o la preferencia por unos u otros tipos de cultivo (Antolín et al., 2015), siempre desde una óptica progresiva micro a macro, y con una escala cronológica clara y válida.
En conclusión, con un aumento exponencial de las fechas radiocarbónicas y de los datos relativos a la cerámica decorada, el papel de Cataluña en el proceso de neolitización del NW mediterráneo cuenta con mejores argumentos para su uso en las discusiones actuales. Se ha comprobado en primer lugar la existencia de diferentes fases a nivel cronológico y sobretodo, como en cada una de ellas se desarrollan diferencias a nivel de producciones cerámicas decoradas en las diferentes regiones analizadas. Establecer el paralelismo entre ceramización y neolitización es arriesgado, sin embargo, en estos momentos es el elemento de la cultura material que traza mejor las diferencias y/o afinidades entre las primeras comunidades agro-ganaderas.

\section{NOTA}

Este artículo es un resumen de la discusión de la tesis doctoral del autor, titulada "La neolitització del nord-est de la Península Ibèrica a partir de les datacions de $14 \mathrm{C}$ i de les primeres ceràmiques impreses $\mathrm{C}$. 5600-4900 cal BC", defendida el 9 de octubre de 2014 en la Universitat de Barcelona. El trabajo fue dirigido por la Dra. M. Àngels Petit Mendizàbal, a quien agradecemos su dedicación y disponibilidad durante todo el proceso de elaboración del mismo.

Damos las gracias a los investigadores que han cedido materiales (cerámicos y/o radiocarbónicos) para el citado trabajo: Araceli Martín, Josep Mestres, Miquel Molist, Anna Gómez, Josep Bosch y Marta Fontanals.

Esta investigación se enmarca en el grupo consolidado SERP (2014SGR-108) y en el proyecto "Substitucions humanes i transformacions econòmiques entre el Pleistocè superior final i l'Holocè al Penedès" 20142017 (2014/100482). 


\begin{tabular}{|c|c|c|c|c|c|c|c|c|}
\hline YACIMIENTO & NIV./ESTR. & Muestra & Referencia & Calidad & BP & Var. & Cal BC $2 \sigma$ & $X^{2}$ \\
\hline \multicolumn{9}{|c|}{ Fase 0} \\
\hline Guixeres Vilobí & A & Ovis/Capra & OxA-26068 & 1 & 6655 & 45 & 5644-5491 & \\
\hline Cavet & 2014 & Triticum a/d & OxA-26061 & 1 & 6536 & 36 & $5610-5386$ & \\
\hline \multicolumn{9}{|c|}{ Fase 1} \\
\hline Cova del Toll & $2 b$ & Ovis/Capra & OxA-26070+26071 & 1 & 6406 & 36 & $5471-5321$ & $3.8495 \%$ \\
\hline Guixeres Vilobí & A & Ovis aries & OxA-26069 & 1 & 6458 & 38 & 5485-5342 & \\
\hline Cavet & 2012 & Triticum a/d & OxA-25802 & 1 & 6440 & 40 & $5479-5331$ & \\
\hline La Serreta & E59 & Angiosperma & Beta-280860 & 2 & 6410 & 40 & $5472-5322$ & \\
\hline La Serreta & E61 & Arbutus $u$. & Beta-280862 & 1 & 6490 & 40 & 5527-5367 & \\
\hline La Serreta & E79 & Arbutus u. & Beta-280866 & 1 & 6420 & 40 & $5473-5326$ & \\
\hline Balma Margineda & $3 b$ & C. avellana & Beta-325682 & 1 & 6410 & 40 & $5472-5322$ & \\
\hline Pça. Vila Madrid & No det. & H. humano & Beta-18271 & 2 & 6440 & 40 & $5479-5331$ & \\
\hline Cova Can Sadurní & C18 & Semillas & Oxa15491+15489+15488 +UBAR760 & 1 & 6396 & 38 & $5470-5316$ & $7.8195 \%$ \\
\hline Can Roqueta II & CR-173 & Ovis aries & Beta-189076 & 1 & 6400 & 50 & 5476-5306 & \\
\hline Balma Serrat Pont & III.4 & Sus scropha & Beta172521 & 2 & 6470 & 40 & $5509-5344$ & \\
\hline Font del Ros & NE 36 & Semilla & AA-16502 & 1 & 6370 & 57 & $5474-5227$ & \\
\hline \multicolumn{9}{|c|}{ Fase 2} \\
\hline Cova Font Major & $\lg$ & Ovis aries & Beta-317705 & 1 & 6310 & 40 & $5367-5215$ & \\
\hline Sant Pau Camp & s. 1 & Ovis aries & Beta-236174 & 1 & 6290 & 50 & $5372-5076$ & \\
\hline Sant Pau Camp & s.2 & Ovis aries & Beta-236175 & 1 & 6250 & 40 & $5316-5070$ & \\
\hline Cova del Frare & C6 & Ovis aries & Beta-325690 & 1 & 6170 & 40 & $5221-5000$ & \\
\hline La Draga & Sector C & Ovis aries & Beta-278255+278256 & 1 & 6220 & 40 & $5302-5057$ & $3.8495 \%$ \\
\hline La Draga & Sector B & Semilla+Bos & OxA20231+20232+Beta & 1 & 6160 & 31 & $5214-5020$ & $5.9995 \%$ \\
\hline La Draga & Sector A & Semilla & OxA-20233 & 1 & 6179 & 33 & $5221-5025$ & \\
\hline La Draga & E-5 Hogar & Semilla & OxA-20234 & 1 & 6127 & 33 & $5210-4981$ & \\
\hline La Draga & E21 Hogar & Semilla & OxA-20235 & 1 & 6143 & 33 & $5210-5001$ & \\
\hline Cova de la Guineu & le & Laurus $n$. & Beta-406998 & 1 & 6140 & 30 & 5209-5002 & \\
\hline Cova Foradada & Ib base & H. humano & Beta-248524 & 2 & 6200 & 40 & $5295-5045$ & \\
\hline Font del Ros & NE 15 & Semilla & AA-15499 & 1 & 6243 & 56 & $5324-5047$ & \\
\hline Font del Ros & NE 33 & Semilla & AA-16501 & 1 & 6307 & 68 & $5468-5075$ & \\
\hline Cova Colomera & CV10 & Buxus s. & Beta-279478 & 1 & 6180 & 40 & $5286-5002$ & \\
\hline Cova Colomera & CE13 & Triticcum a/d & Beta-240551 & 1 & 6150 & 40 & $5216-4993$ & \\
\hline Cova Colomera & CE14 & Triticcum a/d & OxA-23634 & 1 & 6170 & 30 & $5216-5031$ & \\
\hline Cova del Vidre & II hogar & Carbón+Ovis & Oxa26063+Beta58934 & 1 & 6180 & 62 & $5291-4943$ & $3.8495 \%$ \\
\hline Cova del Vidre & $\|$ & Ovis aries & Oxa-26065 & 1 & 6243 & 33 & $5308-5076$ & \\
\hline Cova Sant Llorenç & $\mathrm{n}-2$ & Triticum sp. & Beta-299597 & 1 & 6200 & 40 & $5295-5045$ & \\
\hline Plansallosa & PSL-I & Carbón & Beta-74313+74311 & 2 & 6155 & 60 & $5291-4943$ & $3.8495 \%$ \\
\hline Cova Bonica & IVe FP14 & Arbututs $u$. & OxA-26062 & 1 & 6340 & 34 & $5464-5221$ & \\
\hline Turó Can Bellsolà & TCB-4 & Carbón & AA-19187 & 2 & 6250 & 80 & $5463-4997$ & \\
\hline \multicolumn{9}{|c|}{ Fase 3} \\
\hline La Draga & E3-hogar & Carbón & HD-15451 & 2 & 6060 & 40 & $5195-4842$ & \\
\hline Cova del Parco & EE1 & Carbón & GrN-20058 & 2 & 6120 & 90 & $5297-4839$ & \\
\hline Cova Gran & E9 & Bellota & Beta-265982 & 2 & 6020 & 50 & $5045-4791$ & \\
\hline Coll Blanc & No det. & Carbón & Cedad-no det & 2 & 6009 & 50 & $5037-4783$ & \\
\hline La Draga & E56-hogar & Semillas & UBAR-313 & 1 & 6010 & 70 & $5201-4721$ & \\
\hline Cova Colomera & CE12 & Buxus s. & Beta-248523 & 1 & 6020 & 40 & $5011-4799$ & \\
\hline Balma Margineda & $\mathrm{C} 3 \mathrm{~F}$ & Ovis/Capra & CNA-2681.1 & 1 & 6083 & 38 & $5027-4849$ & \\
\hline Font del Ros & NE 21 & Semilla & AA-16500 & 1 & 6058 & 79 & $5211-4790$ & \\
\hline
\end{tabular}




\begin{tabular}{|c|c|c|c|c|c|c|c|c|}
\hline \multicolumn{9}{|c|}{ Dataciones reducidas en los $X^{2}$} \\
\hline Cova del Toll & $2 b$ & Ovis/Capra & OxA-26070 & 1 & 6425 & 35 & $5510-5310$ & \\
\hline Cova del Toll & $2 b$ & Ovis/Capra & OxA-26071 & 1 & 6390 & 34 & $5490-5290$ & \\
\hline Cova Can Sadurní & $\mathrm{C} 18$ & Triticcum d. & OxA-15491 & 1 & 6375 & 34 & $5480-5280$ & \\
\hline Cova Can Sadurní & C18 & Triticcum d. & OxA-15489 & 1 & 6391 & 34 & $5490-5290$ & \\
\hline Cova Can Sadurní & C18 & Semillas & UBAR-760 & 1 & 6405 & 50 & $5510-5270$ & \\
\hline Cova Can Sadurní & C18 & Triticcum d. & OxA-15488 & 1 & 6421 & 34 & $5510-5310$ & \\
\hline La Draga & Sector B & Bos taurus & Beta-no det & 1 & 6184 & 27 & $5240-5040$ & \\
\hline La Draga & Sector B & Semilla & OxA-20231 & 1 & 6163 & 33 & $5250-5010$ & \\
\hline La Draga & Sector B & Semilla & OxA-20232 & 1 & 6121 & 33 & $5250-4930$ & \\
\hline La Draga & Sector C & Ovis aries & Beta-278255 & 1 & 6270 & 40 & $5340-5180$ & \\
\hline La Draga & Sector C & Ovis aries & Beta-278256 & 1 & 6170 & 40 & $5250-5010$ & \\
\hline Cova del Vidre & II hogar & Ovis aries & Oxa-26064 & 1 & 6181 & 35 & $5250-5010$ & \\
\hline Cova del Vidre & II hogar & Carbón & Beta-58934 & 2 & 6180 & 90 & $5380-4900$ & \\
\hline Plansallosa & PSL-I & Carbón & Beta-74313 & 2 & 6130 & 60 & $5270-4910$ & \\
\hline Plansallosa & PSL-I & Carbón & Beta-74311 & 2 & 6180 & 60 & $5290-4970$ & \\
\hline
\end{tabular}

Apéndice / Appendix: Dataciones aceptadas para el NE peninsular. Calibración mediante el software OxCal v.4.2.4 y la curva IntCal'13. Datos extraídos de Martins et al., 2015; Oms et al., 2014; Cebria et al., 2014; Pou et al., 2010; Carlus et al., 2008; Alcalde et al., 2002; Pallares et al., 1997; Molist et al., 2008; Bosch et al., 2011; Cebria et al., 2011; Oms et al., 2012; Borrell et al., 2014; Morales et al., 2010; Petit, 1996; Mora et al., 2011; Bravo et al., $2014 ;$ Bosch et al., 1998 Accepted dates from NE Iberia. Calibration through OxCal v.4.2.4 software and IntCal'13 atmospheric curve. Data from Martins et al., 2015; Oms et al., 2014; Cebria et al., 2014; Pou et al., 2010; Carlus et al., 2008; Alcalde et al., 2002; Pallares et al., 1997; Molist et al., 2008; Bosch et al., 2011; Cebria et al., 2011; Oms et al., 2012; Borrell et al., 2014; Morales et al., 2010; Petit, 1996; Mora et al., 2011; Bravo et al., 2014; Bosch et al., 1998.

\section{BIBLIOGRAFIA}

Alcalde, G., Molist, M., Saña, M. (Eds.), 2002. Procés d'ocupació de la bauma del Serrat del Pont (la Garrotxa) entre 5480 2900 cal AC. Museu Comarcal de la Garrotxa. Olot. Publicacions Eventuals d'Arqueologia de la Garrotxa 7.

Alday, A., 2009. Reflejos del Neolítico Ibérico. La cerámica boquique: caracteres,cronología y contexto. EDAR, Barcelona.

Alday, A., Moral del Hoyo, S., 2011. El dominio de la cerámica boquique: discusiones técnicas y cronoculturales. In: Bernabeu Auban, J., Rojo Guerra, M., Molina Balaguer, LI. (Eds.), Las primeras producciones cerámicas. EI VI milenio cal a.C. en la Península Ibérica, 65-80, València. Sagvntvm-PLAV, Extra-12.

Antolín, F., Jacomet, S., Buxó, R., 2015. The hard knock life. Archaeobotanical data on farming practies during the Neolithic (5400-2300 cal BC) in the NE of the Iberian Peninsula. Journal of Archaeological Science 61, 90-104.

Baldellou, V., 1972. El yacimiento prehistórico del Roc d'en Sardinyà (Sant Genís de Vilassar, Barcelona). Pyrenae 8, 41-52.

Baldellou, V., 1974. Los materiales arqueológicos de la Cova Bonica de Vallirana (Barcelona). Ampurias 36, 1-19.

Baldellou, V., 1979. Excavaciones arqueológicas en la Cova del Bolet (Mediona, Barcelona). Pyrenae 15-16, 61-114.

Baldellou, V., Mestres, J., Martí, B., Juan-Cabanilles, J., 1989. El Neolítico Antiguo. Los primeros agricultores y ganaderos en Aragón, Cataluña y Valencia. Diputación de Huesca, Huesca.

Baldellou, V., Utrilla, P., 1999. Le Néolithique en Aragon. In: Vaquer, J. (Dir.), XXIVè Congrès Préhistorique de France, Le Néolithique du Nord-Ouest méditerranéen, Carcassonne 1994, 225-237.

Bernabéu, J., 1989. La tradición cultural de las cerámicas impresas en la zona oriental de la Península Ibérica. Servei d'Investigacions Prehistòriques. València. Serie de Trabajos Varios 86.
Bernabéu, J., 1999. Pots, symbols and territories: the archaeological context of neolithisation in Mediterranean Spain. Documenta Praehistorica XXVI, 101-118.

Bernabéu, J., 2006. Una visión actual sobre el origen y difusión del Neolítico en la Península Ibérica c. 5600-5000 cal BC. In: García, O., Aura, J.E. (Dirs.), El Abric de la Falguera (Alcoi, Alacant). 8000 años de ocupación humana en la cabecera del río Alcoi, 189-211, Ajuntament d'Alcoi-Diputació d'Alacant, C.A.M.

Bernabéu, J., Barton, C., Pérez-Ripoll, M., 2001. A taphonomic perspective on Neolithic beginnings: theory, interpretation and empirical data in the western Mediterranean. Journal of Archaeological Science 28(6), 597-612.

Bernabéu, J., Gómez, O., Molina, LI., García Borja, P., 2011. La cerámica neolítica durante el VI milenio cal $\mathrm{AC}$ en el Mediterráneo central peninsular. In: Bernabéu, J., Rojo, M.A., Molina, LI. (Coords.), Las primeras producciones cerámicas: el VI milenio cal AC en la Península Ibérica, 153-178, Universitat de València Saguntum PLAV Extra-12.

Bernabéu, J., Martí, B., 2014. The first agricultural groups in the Iberian Peninsula. In: Manen, C., Perrin, T., Guilaine, J. (Eds.), La transition néolithique en Mediterranée. The Neolithic transition in the Mediterranean, 419-438, Ed. Érrance- AEP.

Bernabéu, J., Molina, LI., 2009. La Cova de les Cendres (Moraira-Teulada, Alicante). MARQ. Diputació d'Alacant. Serie Mayor, 6.

Bernabéu, J., Molina, LI., Esquembre, M.A., Ortega, J.R., Boronat, J., 2009. La cerámica impresa mediterránea en el origen del Neolítico de la Península Ibérica? In: De Méditerranéen et d'ailleurs... Mèlanges offerts à Jean Guilaine, 83-95, Archives d'Ecologie Préhistorique, Toulouse.

Bernabéu, J., Rojo, M.A., Molina, LI., 2011. Las primeras producciones cerámicas: el VI milenio cal AC en la Península Ibérica. Saguntum PLAV Extra-12, Universitat de València. 
Bernabo Brea, L., 1949. La cultura prehistoriche della Francia Meridionale e della Catalogna e la successione stratigrafica delle Arene Candide. Revue d'Études Ligures XV(1-2), 21-45.

Binder, D., 2013. Mésolithique et Néolithique ancien en Méditerranée nord-occidentale entre 7000 et 5500 cal. BC: questions ouvertes sur les dynamiques culturelles et les procès d'interaction. In: Jaubert, J., Fourment, N., Depaepe, P. (Dirs.), XXVIle Congrès Préhistorique de France - Session Transitions, ruptures et continuité en Préhistoire, Bordeaux-Les Eyzies, 31 mayo-5 junio de 2010, 341-355.

Binder, D., Brochier, J.E., Duday, H., Helmer, D., Marinval, P., Thiébault, S., Wattez, J., 1993. L'abri Pendimoun à Castellar (Alpes-Maritimes). Nouvelles donnés sur le complexe culturel de la céramique imprimée méditerranéenne dans son contexte stratigraphique. Gallia Prehistoire 35, 177-255.

Binder, D., Clop, X., Convertini, F., Manen, C., Sénépart, I., 2010. Les productions céramiques du Néolithique ancien entre Provence et Catalogne. In: Manen, C., Convertini, F., Binder, D., Sénépart, I. (Dirs.), Premières societés paysannes de Méditerrannée occidental. Structures des productions céramiques, 115-132, Société Préhistorique Française, Toulouse. Mémoire 51.

Binder, D., Maggi, R., 2001. Le Néolithique ancien de l'arc liguro-provençal. Bulletin de la Société Préhistorique Française 98(3), 411-422.

Binder, D., Sénépart, I., 2010. La séquence de l'Impresso-Cardial de l'abri Pendimoun et l'évolution des assemblages céramiques de la Provence. In: Manen, C., Convertini, F., Binder, D., Sénépart, I. (Dirs.), Premières societés paysannes de Méditerrannée occidental. Structures des productions céramiques, 149-167, Société Préhistorique Française, Toulouse. Mémoire 51.

Borrell, F., Gómez, A., Molist, M., Tornero, C., Vicente, O.. 2014. Les ocupacions de la cova de Sant Llorenç (Sitges, Garraf): Noves aportacions al coneixement de la prehistòria del Garraf. Tribuna d'Arqueologia 2011-2012, 110-128.

Bosch, À., Buxó, R., Palomo, A., Buch, M., Mateu, J., Tabernero, E., Casadevall, J., 1998. El poblat neolític de Plansallosa. L'explotació del territori dels primers agricultors ramaders de l'Alta Garrotxa. Publicacions Eventuals d'Arqueologia de la Garrotxa 5.

Bosch, À., Chinchilla, J., Tarrús, J., 2000. El poblat lacustre neolític de la Draga. Excavacions de 1990 a 1998. Monografies del CASC 3.

Bosch, À., 2011. El poblat lacustre del neolític antic de la Draga. Excavacions 2000-2005. Girona. Monografies del CASC 9.

Bosch, J., 2015. La cueva del Vidre (Roquetes, bajo Ebro). Asentamiento del Mesolítico y Neolítico antiguo en la cordiIlera litoral meridional catalana. In: Gonçalvez, V.S., Diniz, M., Sousa, A.C. (Coords.), V Congresso de Neolítico Peninsular, Lisboa, abril 2011, 182-188.

Bosch Gimpera, P., 1915-1920. Les coves properes a la costa catalana, Crònica de la Secció d'Arqueologia de l'Institut d'Estudis Catalans. Anuari de I'Institut d'Estudis Catalans VI, 476-477.

Bravo, P., Garcia, M., Solà, E., 2014. Darreres aportacions al coneixement del neolític i l'edat del bronze al Camp de Tarragona: intervencions als terrenys de l'aeroport de Reus i a la partida de Coll Blanc (Reus, Baix Camp). Tribuna d'Arqueologia 2011-2012, 173-188.

Briois, F., Manen, C., 2009. L'habitat néolithique de Peiro Signado à Portiragnes. In: Beeching, A. \& Sénépart, I. (Dirs.), De la maison au village. L'habitat néolithique dans le Sud de la France et le Nord-Ouest méditerranéen, 31-38, Mémoire de la Société Préhistorique Française 48.
Bronk Ramsey, C., 2013. OxCal 4.2.2 Manual. Available from: https://c14.arch.ox.ac.uk.

Carlús, X., López-Cachero, J., Terrats, N., Oliva, M., Palomo, A., Rodríguez, A., 2008. Diacronia durant la prehistòria recent a Can Roqueta (Sabadell-Barberà del Vallès; Vallès Occidental) entre el VI i el I mil·lenni cal BC. Cypsela 18, 115-142.

Carvalho, A.F., 2008. A Neolitização do Portugal Meridional. Os exemplos do Maciço Calcário Estremenho e do Algarve Ocidental. Universidade do Algarve. Promontoria Monográfica 12.

Carvalho, A.F., 2010. Chronology and geography of the Mesolithic-Neolithic transition in Portugal. In: Henning, J., Leube, A., Biermann, F. (Eds.). On Pre- and Earlier History of Iberia and Central Europe. 45-61. Habelt-Verlag, Bonn. Studien zur Archäologie Europas 11.

Cebrià, A., Fontanals, M., Martín, P., Morales, J.I., Oms, F.X., Rodríguez Hidalgo, A., Soto, M., Vergés, J.M., 2014. Nuevos datos para el Neolítico antiguo del NE peninsular procedentes de la Cova del Toll (Moià, Barcelona) y de la Cova de la Font Major (L'Espluga de Francolí, Tarragona). Trabajos de Prehistoria $71(1), 137-148$.

Ibáñez, J.J., Gibaja J.F., Gassin, B., Mazzuco, N., 2015. Les premières faucilles et l'expansion de l'agriculture en Europe. ArchéOrient - Le Blog (Hypotheses.org), 6 mars 2015. Available from: http://archeorient.hypotheses.org/3788.

Colomines, J., 1925. Prehistoria de Montserrat. Analecta Montserratina VI, 225-349.

Colomines, J., 1947. La Cueva de Can Montmany de Pallejà. Empúries 9-10, 237-242.

D.D.A.A. 1915-1920. Resultats de l'exploració de coves de Catalunya, Crònica de la Secció d'Arqueologia de I'Institut d'Estudis Catalans. Anuari de l'Institut d'Estudis Catalans VI, 473-491.

D.D.A.A., 1981. El neolític a Catalunya. Taula rodona de Montserrat, mayo 1980.

Defranould, E., 2012. Essai de sériation chronoculturelle du Néolithique ancien: apport des industries lithiques de la Baume de Ronze, Orgnac-l'Aven, Ardèche. Université de Toulouse le Mirail. Memoria de Máster 2 Inédita.

Edo, M., Blasco, A., Villaba, M.J., 2011. Guió sintètic de la prehistòria recent de Garraf. In: Blasco, A., Edo, M., Villalba, M.J. (Coords.), La Cova de Can Sadurní i la prehistòria de Garraf. Recull de 30 anys d'investigació, 13-96, Ed. EDAR, Begues.

García Atiénzar, G., 2010. Las comarcas centromeridionales valencianas en el contexto de la Neolitización de la fachada noroccidental del Mediterráneo. Trabajos de Prehistoria 67(1), 37-58.

García Borja, P., Aura, E., Jordà Pardo, J.F., Salazar, D.C., 2014. La cerámica neolítica de la Cueva de Nerja (Málaga, España): salas del Vestíbulo y la Mina. Archivo de Prehistoria Levantina XXX, 81-31.

Garcia Borja, P., Cortell, E., Pardo, S., Pérez, G., 2011a. Las cerámicas de la Cova de l'Or (Beniarrés, Alacant). Tipología y decoración de las colecciones del Museu d'Alcoi. Recerques del Museu d'Alcoi 20, 71-136

Garcia Borja, P., Martins, H., Cortell, S., Pardo, S., 2012. Dataciones radiocarbónicas en contextos del Neolítico antiguo de la Cova Fosca de la Vall d'Ebo (Alacant, España). Alberri 22, 78-97.

Garcia Borja, P., Salazar, D., Pérez, A., Pardo, S., Casanova, V., 2011b. El Neolítico antiguo cardial y la Cova de la Sarsa (Bocairent, Valencia). Nuevas perspectivas a partir de su registro funerario. Munibe Antropologia-Arkeologia 62, 175-195. 
García Martínez de Lagrán, I., 2014. La neolitización de la Meseta norte y de la alta y media cuenca del Ebro (España): premisas teóricas, análisis del registro y planteamiento de hipótesis. Zephyrus LXXIII, 83-107.

García Martínez de Lagrán, I., Garrido, R., Rojo, M.A., Alday, A., García Gazólaz, J., Sesma, J., 2011. Cerámicas, estilo y neolitización: estudio comparativo de algunos ejemplos de la Meseta norte y el alto valle del Ebro. In : Bernabéu, J., Rojo, M.A., Molina, L.I. (Coords.), Las primeras producciones cerámicas: el VI milenio cal AC en la Península Ibérica. 83-104. Universitat de València. Saguntum PLAV Extra-12.

García-Puchol, O., Aura, J.E., 2006. El Abric de la Falguera (Alcoi, Alacant). 8000 años de ocupación humana en la cabecera del río Alcoi. Ajuntament d'Alcoi-Diputació d'Alacant, C.A.M.

Giró, P., 1947-1948. Cuevas del Bolet (Mediona). Ampurias IXX, 261. GRIVÉ, J. 1936 L'Esquerda de les Roques del Pany (Penedès). Anuari de I'Institut d'Estudis Catalans VIII (19271931), 19-33.

Grivé, J., 1936. L'Esquerda de les Roques del Pany (Penedès). Anuari de l'Institut d'Estudis Catalans VIII (1927-1931), 19-33

Guilaine, J., 1976. Premiers bergers et paysans de l'Occident méditerranéen. Ed Mouton. Paris.

Guilaine, J., 2007. Du concept de céramique imprimée méditerranéenne à la genèse du Cardial. In: Guilaine, J., Manen, C., Vigne, J.D. (Dirs.), Pont de Roque-Haute, Nouveaux regards sur la néolithisation de la France méditerranéenne, 21-38, Toulouse.

Guilaine, J., Barbaza, M., Gasco, J., Geddes, D., Coularou, J., Vaquer, J., Brocher, J.E., Briois, F., Andre, J., Jalut, G., Vernett, J.L., 1993. Dourgne, derniers chasseurs-collecteurs et premiers éleveurs de la Haute-Vallée de l'Aude. Centre d'Anthropologie des Sociétés Rurales, EHESS. Toulouse.

Guilaine, J., Barbaza, M., Geddes, D., Vernet, J.L., Llongueras, M., Hopf, M., 1982. Prehistoric human adaptations in Catalonia (Spain). Journal of Field Archaeology 9, 408-416.

Guilaine, J., Freises, A., Montjardin, R., 1984. Leucate-Corrège. Habitat noyé du Néolithique Cardial. Centre d'Anthropologie des Sociétés Rurales, EHESS, Toulouse.

Guilaine, J., Gascó, J., Vaquer, J., Barbaza, M., 1979. L’abri Jean Cros: essai d'approche d'un groupe humain du Néolithique ancien dans son environnement. Centre d'anthropologie des sociétés rurales, Toulouse.

Guilaine, J., Manen, C., 2007. From Mesolithic to Early Neolithic in the western Mediterranean. Proceedings of the British Academy 144, 21-51.

Guilaine, J., Manen, C., Vigne, J.D., 2007. Pont de Roque-Haute. Noveaux regards sur la néolithisation de la France méditerranéene. EHESS-CRPPM, Toulouse. Archives d'Écologie Préhistorique.

Guilaine, J., Van Willigen, S., Convertini, F., 2008. La céramique du Néolithique ancien de la grotte du Pont à Poussarou (Hérault). Bulletin de la Société préhistorique française 105(4), 749-771.

Juan-Cabanilles, J., Martí, B., 2002. Poblamiento y procesos culturales en la Península Ibérica del VII al $\mathrm{V}$ milenio a.C. (8000-5500 BP). Una cartografía de la neolitización. In : Bernabéu, J., Badal, E., Martí, B. (Eds), El paisaje en el Neolítico Mediterráneo, 45-87, Saguntum PLAV Extra 5.

Le Bras-Goude, G., Binder, D., Zemour, A., Richards, M., 2010. New radiocarbon dates and isotopic analyses of Neolithic human and animal bone from the Fontbrégoua cave (Salernes, Var, France). Journal of Anthropological Sciences 88, 167-178.
Manen, C., 2000. Le Néolithique ancien entre Rhône et Ebre: analyse des céramiques décorées. EHESS, Toulouse. Tesis doctoral inédita.

Manen, C., 2002. Structure et identité des styles céramiques du Néolithique ancien entre Rhône et Ėbre. Gallia Préhistoire 44, 121-166.

Manen, C., 2007. La production céramique de Pont de Roque-Haute: synthése et comparisons. In: Guilaine, J., Manen, C., Vigne, J.D. (Dirs.), Pont de Roque-Haute. Noveaux regards sur la néolithisation de la France méditerranéene, 151-166, EHESS-CRPPM, Toulouse. Archives d'Écologie Préhistorique.

Manen, C., Convertini, F., Binder, D., Sénépart, I., 2010a. Premières sociétés paysannes de Méditerranée occidental. Structures des productions céramiques. Société Préhistorique Française, Toulouse. Mémoire 51.

Manen, C., Guilaine, J., 2010. Aspects géographiques et chronoculturels du Néolithique ancien languedocien. In: Manen, C., Convertini, F., Binder, D., Sénépart, I. (Dirs.), Premières sociétés paysannes de Méditerranée occidental. Structures des productions céramiques, 179-190, Société Préhistorique Française, Toulouse. Mémoire 51.

Manen, C., Sabatier, P., 2003. Chronique radiocarbone la néolithisation en Méditerranée nord-occidental. Bulletin de la Société préhistorique Française 100(3), 479-504.

Manen, C., Salanova, L., 2010. Les impressions de coquilles marines à front denté dans les décors céramiques néolithiques. In: Manen, C., Convertini, F., Binder, D., Sénepart, I. (Dirs.), Premières sociétés paysannes de Méditerranée occidental. Structures des productions céramiques, 57-64, Société Préhistorique Française, Toulouse. Mémoire 51.

Manen, C., Sénépart, I., Binder, D., 2010b. Les productions céramiques des groupes cardiaux et épicardiaux du Sud de la France: zoom regional. In: Manen, C., Convertini, F., Binder, D., Sénépart, I. (Dirs.), Premières societés paysannes de Méditerrannée occidental. Structures des productions céramiques, Toulouse, 11-12 mai 2007, 191-196, Société Préhistorique Française, Paris. Mémoire 51.

Manen, C., Vigne, J.D., Loirat, D., Boudy, L., 2001. L'Aspre del Paradis à Corneilla-del-Vercol (Pyrennées-Orientales): contribution à l'étude du Néolithique ancien et final roussillonnais. Bulletin de la Société Préhistorique française 98(3), 505-528

Martí, B., 1977. Cova de l'Or (Beniarrés, Alicante). Diputació de Valencia, Servicio de Investigación Prehistórica, València. vol. 1.

Martí, B., 2008. Cuevas, poblados y santuarios neolíticos: una perspectiva mediterránea. In: Hernández, M.S., Soler, J.A., López Padilla, J.A. (Eds.), IV Congreso del Neolítico Peninsular, vol. 1, 17-27, MARQ, Alicante.

Martí, B., 2011. Cova de l'Or (Beniarrés, Alacant). In: Bernabéu, J., Rojo, M.A., Moina, LI. (Eds.), Las primeras producciones cerámicas: el VI milenio cal AC en la Península Ibérica, 183-186, Universitat de València. Saguntum PLAV Extra-12.

Martín, A., 1992. Dinámica del Neolítico antiguo y medio en Cataluña. In: Utrilla, P. (Dir.), Aragón/Litoral mediterráneo: intercambios culturales durante la Prehistoria, Coloquio de Zaragoza, 319-333, Institución Fernando el Católico, Zaragoza.

Martin, A., 2000. Els primers pastors de Sant Llorenç del Munt al Vlè mil.lenni aC. In: IV Trobada d'Estudiosos de Sant Llorenç del Munt i l'Obac, Barcelona. Diputació de Barcelona, 197-204.

Martín, A., Edo, M., Tarrus, J., Clop, X., 2010. Le Néolithique ancien de Catalogne ( $\mathrm{VI}$ - première moitié du $\mathrm{V}$ millénaire av. J.C.). Les séquences chronoculturels. In: Manen, C., Convertini, F., Binder, D., Sénépart, I. (Dirs.), Premières societés pay- 
sannes de Méditerrannée occidental. Structures des productions céramiques, 197-214, Société Préhistorique Française, Toulouse. Mémoire 51.

Martín, A., Vaquer, J., 1995. El poblament dels Pirineus a I'Holocè, del mesolític a l'edat del bronze. In: Bertranpetit, J., Vives, E. (Eds), Muntanyes i població. El passat del Pirineus des una perspectiva multidisciplinària, 35-73, Andorra la Vella.

Martins, H., Oms, F.X., Pereira, L., Pike, A., Rowsell, K., Zilhão, J., 2015. Radiocarbon dating the beginning of the Neolithic in Iberia: New results, new problems. Journal of Mediterranean Archaeology 28(1), 105-131.

Mestres, J., 1981-1982. Avançament a l'estudi del jaciment de les Guixeres de Vilobí. Pyrenae 17-18, 35-53.

Mestres, J., 1992. Neolitització i territori, en 9è Col-loqui Internacional d'Arqueologia de Puigcerdà. Estat de l'investigació sobre el Neolític a Catalunya 1991, 72-75, Puigcerdà y Andorra la Vella

Molist, M., Vicente, O., Farré, R., 2008. El jaciment de la Caserna de Sant Pau: aproximació a la caracterització d'un assentament del Neolític antic. QUARHIS 4, 14-24.

Montjardin, R., Cablat, A., 2004. Le gisement Néolithique ancien des Dunes à Frontignan (Hérault). In: Dartevelle, H. (Dir.), Auvergne et Midi. Actualité de la recherche, actes des 5es Rencontres méridionales de Préhistoire récente (Clermont-Ferrand, 2002), Cressensac, Préhistoire du Sud-Ouest, 295-312, Supplément à Préhistoire du Sud-Ouest 9.

Navarrete, M.S., 1976. La cultura de las cuevas con cerámica decorada en Andalucía Oriental. Universidad de Granada.

Oms, F.X., 2014. La neolitització del nord-est de la Península Ibèrica a partir de les datacions de $14 \mathrm{C}$ i de les primeres ceràmiques impreses c. 5600-4900 cal BC. Universitat de Barcelona. Tesis doctoral, 479p.

Oms, F.X., Esteve, X., Mestres, J., Martín, P., Martins, H., 2014. La neolitización del nordeste de la Península Ibérica: datos radiocarbónicos y culturales de los asentamientos al aire libre del Penedès. Trabajos de Prehistoria 71(1), 43-56.

Oms, F.X., Cebrià, A., Morales, J.I., Pedro, M., 2016a. Una inhumació cardial a la cova Foradada (Calafell, Baix Penedès). In: Jornades d'Arqueologia del Penedès octubre 2011. Institut d'Estudis Penedesencs. Vilafranca del Penedès, 117-134.

Oms, F.X., Gibaja, J.F., Mazzuco, N., Guilaine, J., 2016b. Revisión radiocarbónica y cronocultural del Neolítico antiguo de la Balma Margineda (Aixovall, Andorra). Trabajos de Prehistoria 73(1), 29-46.

Oms, F.X., Martín, A., Esteve, X., Mestres, J., Morell, B., Gibaja, J.F., 2016c. The Neolithic in North-East Iberia: Chronocultural Phases and 14C. Radiocarbon 58(2), 291-309.

Oms, F.X., Petit, M.A., Morales, J.I., García, M.S., 2012. Le processus de néolithisation dans les Pyrénées orientales. Occupation du milieu, culture matérielle et chronologie. Bulletin de la Societé Préhistorique Française 109(4), 651-670.

Pallarés, M., Bordas, A., Mora, R., 1997. El proceso de neolitización en los Pirineos Orientales. Un modelo de continuidad entre los cazadores-recolectores neolíticos y los primeros grupos agropastoriles. Trabajos de Prehistoria 54(1), 121-141.

Polo, A., Martínez-Moreno, J., Benito-Calvo, A., Mora, R., 2014. Prehistoric herding facilities: site formation and archaeological dynamics in Cova Gran de Santa Linya (Southeastern Prepyrenees, Iberia). Journal of Archaeological Science 41, 1-17. http://dx.doi.org/10.1016/j. jas.2013.09.013.
Pou, R., Martí, M., Jordana, X., Malgosa, A., Gibaja, J.F., 2010. L'enterrament del Neolític antic de la Plaça Vila de Madrid (Barcelona): una estructura funeraria del Vlè mil·lenni $\mathrm{aC}$. QUARHIS 6, 94-107.

Ramón, N., 2006. La cerámica del Neolítico Antiguo en Aragón. Caesaragusta 77, 9-353.

Reime, P.J., Bard, E., Bayliss, A., Beck, J.W., Blackwell, P.G., Bronk Ramsey, C., Buck, C.E., Cheng, H., Edwards, R.L., Friedrich, M., Grootes, P.M., Guilderson, T.P., Haflidason, H., Hajdas, I., Hatté, C., Heaton, T.J., Hoffmann, D.L., Hogg, A.G., Hughen, K.A. Kaise, K.F., Kromer, B., Mannining, S.W., Niu, M., Reimer, R.W., Richards, D.A., Scott, M., Southon, J.R., StaffF, R.A., Turney, C.S.M., Van Der Plicht, J., 2013. Intcal13 and Marine13 radiocarbon age calibration curves $0-50,000$ years cal BP. Radiocarbon 55: 1869-1887.

Rojo, M.A., Kunst, M., Garrido, R., García, I., Morán, G., 2008. Paisajes de la memoria: asentamientos del Neolítico antiguo en el Valle de Ambrona (Soria, España). Universdad de Valladolid, Instituto Arqueológico Alemán.

Rojo, M.A., Peña-Chocarro, L., Royo, J.I., Tejedor, C., García Martínez de Lagrán, I., Arcusa, H., Garrido, R., Moreno-García, M., Mazzuco, N., Gibaja, J.F., Ortega, D., Kromer, B., Alt, K.W., 2013. Pastores transhumantes del Neolítico antiguo en un entorno de alta montaña: secuencia crono-cultural de la Cova de Els Trocs (Sant Feliú de Veri, Huesca). BSAA Arqueología LXXIX, 9-58.

Rojo, M.A., Tejedor, C., Peña-Chocarro, L., Royo, J.I., García Martínez de Lagrán, I., Arcusa, H., San Millán, M., Garrido, R., Gibaja, J.F., Mazzuco, N., Clemente, I., Mozota, M., Terradas, X., Moreno-García, M., Pérez-Jordá, G., Álvarez-Fernández, E., Jiménez, I., Gómez, F., 2015. Releyendo el fenómeno de la Neolitización en el Bajo Aragón a la luz de la excavación del Cingle de Valmayor XI (Mequinenza, Zaragoza). Zephyrus LXXV, 41-71.

Roudil, J.-L., Van Willingen, S., 2002. La céramique du Néolithique ancien de la Baume d'Oullins (Le Garn, Gard). Ardèche Archéologie 19, 6-26.

Serra Ràfols, J. de C., 1921. La col-lecció arqueològica Lluís Marian Vidal. Publicacions del Seminari de Prehistòria de la Universitat de Barcelona, Barcelona.

Soler, J.A., Duque, D., Ferrer, C., García Atiénzar, G., Gómez, O., Guillem Calatayud, P., Iborra, P., Martínez Valle, R., Pérez Jordà, G., Roca de Togores, C., Ximénez de Embún, T., 2013. Sobre el significado de la primera ocupación neolítica de la Cova d'en Pardo (Planes, Alicante): avance de estudio pluridisciplinar de los niveles VIII y VIIIB. Saguntum PLAV 45, 9-24.

Tarrús, J., 1981. El Neolític antic a les comarques gironines, a El Neolític a Catalunya, Taula Rodona de Montserrat 1980, 3358, Publicacions de l'Abadia de Montserrat.

Torregrossa, P., Jover, F., López, E., 2011. Benàmer (Muro d'Alcoi, Alicante). Mesolíticos y neolíticos en las tierras meridionales valencianas. Diputació de València SIP. Serie de Trabajos Varios 112.

Utrilla, P., 2012. Caminos para el neolítico aragonés. La aportación del radiocarbono y del arte rupestre. In: Congrés Internacional Xarxes al Neolitic, Gavá-Bellatera 2011, 555-563.

Utrilla, P., Domingo, R., 2014. La transition Mésolithique-Néolithique dans la vallée de l'Ebre. In: Manen, C., Perrin, T., Guilaine, J. (Eds.). La transition néolithique en Mediterranée. The Neolithic transition in the Mediterranean, 327-358, Érrance - AEP.

Van Willingen, S., 2004. Aspects culturels de la néolithisation en Méditerranée occidentale: le Cardial et l'Épicardial. Bulletin de la Société Préhistorique Française 101(3), 463-495. 
Van Willingen, S., Hajdas, I., Bonani, G., 2009. New radiocarbon dates for the early Neolithic of the western Mediterranean. Radiocarbon 51(2), 831-838.

Vilaseca, S., 1969. La Cova de la Font Major. Trabajos de Prehistoria XXVI, 117-202.

Zilhão, J., 2001. Radiocarbon evidence for maritime pioneer colonisation at the origins of farming in west Mediterranean Europe. PNAS 98(24), 14180-14185

Zilhão, J., 2011. Time is on my side... In: Hadjikoumis, A., Robinson, E., Viner, S. (Eds), The dynamics of Neolithisation in Europe. Studies in honour of Andrew Sherrat, 46-65, Oxbow books. 
